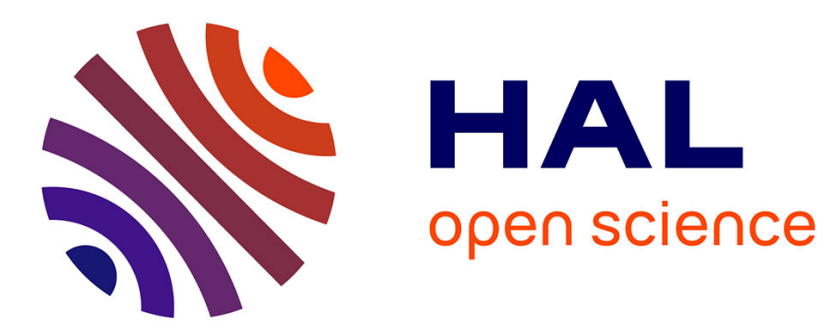

\title{
Mathematical analysis of the optimal habitat configurations for species persistence
}

Lionel Roques, François Hamel

\section{To cite this version:}

Lionel Roques, François Hamel. Mathematical analysis of the optimal habitat configurations for species persistence. 2007. hal-00149538

\section{HAL Id: hal-00149538 \\ https://hal.science/hal-00149538}

Preprint submitted on 28 May 2007

HAL is a multi-disciplinary open access archive for the deposit and dissemination of scientific research documents, whether they are published or not. The documents may come from teaching and research institutions in France or abroad, or from public or private research centers.
L'archive ouverte pluridisciplinaire HAL, est destinée au dépôt et à la diffusion de documents scientifiques de niveau recherche, publiés ou non, émanant des établissements d'enseignement et de recherche français ou étrangers, des laboratoires publics ou privés. 


\title{
Mathematical analysis of the optimal habitat configurations for species persistence
}

\author{
Lionel Roques and François Hamel
}

May 28, 2007

\begin{abstract}
We study a reaction-diffusion model in a binary environment made of habitat and non-habitat regions. Environmental heterogeneity is expressed through the species intrinsic growth rate coefficient. It was known that, for a fixed habitat abundance, species survival depends on habitat arrangements. Our goal is to describe the spatial configurations of habitat that maximise the chances of survival. Through numerical computations, we find that they are of two main types - ball-shaped or stripe-shaped. We formally prove that these optimal shapes depend on the habitat abundance and on the amplitude of the growth rate coefficient. We deduce from these observations that the optimal shape of the habitat realises a compromise between reducing the detrimental habitat edge effects and taking advantage of the domain boundary effects. In the case of an infinite-periodic environment, we prove that the optimal habitat shapes can be deduced from those in the case of a bounded domain.
\end{abstract}

\section{Introduction}

In the recent literature, the loss of habitat connectivity, also known as environmental fragmentation, has been more and more often cited as a major cause of species endangerment. In the IUCN Red List of Endangered Species [1], it is shown to be one of the most important threats on the endangered species.

The main causes of these habitat losses and fragmentation are related to human activities. For instance, roads create barriers for many animals, and carnivores are particularly vulnerable to the resulting habitat fragmentation (see e.g. Treves et al. [2]). Agricultural and forestry activities are the key drivers of habitat loss affecting birds [1]. In freshwater, dam construction is a well-established cause of ecosystem fragmentation and species extinction (an example is given by Morita and Yamamoto [3]). Habitat fragmentation can also be the result of climate changes. Indeed warming up can reduce the size of the habitat patches for some mountain-top species; it is also responsible for the shrinking of lakes and rivers, which may disconnect sub-populations.

Though it seems natural that habitat loss is a major threat to species persistence, the effects of habitat fragmentation per se, i.e., without habitat loss, 
depend on the considered ecosystem. Nevertheless, most theoretical studies involving single-species models (see below for details and references) predict a negative impact of fragmentation on species persistence. Indeed, the larger the habitat patches are, the more likely the sub-populations will be important and therefore less susceptible to internal and external extinction factors. These theoretical results are supported experimentally for some animals and plants [4, 5]. However, a recent analysis of several empirical studies, by Fahrig [6], shows that fragmentation per se can also have positive effects on biodiversity, which is notably explained in her paper by interspecific interactions. The long-standing debate on "single large or several small" reserves (see Simberloff [7] for a discussion and further references on this subject) illustrates the complexity of the issue of the effects of habitat fragmentation.

In addition to the number and area of the habitat patches, their shape can also have an influence on species persistence. For some species, the habitat's edge has a detrimental effect, for instance because of increased predation, or increased risk of accident (see Chalfoun et al. [8] and Lovejoy et al. [9]). Stripe shaped habitat patches have large edge/area ratios, compared to round shaped habitat patches. Thus, it seems natural to claim, like Diamond [10], that species for which the edge has a detrimental effect will be more likely to survive in the latter case of round shaped habitat patches.

The important question of the effects of habitat fragmentation has stimulated the development of numerous population models taking account of environmental heterogeneity. In individual based models (IBM) each individual interacts with its environment, and simulations are run until a general behaviour of the population can be observed. Typically, with this approach, With and King [11] showed that the probability of dispersal success increased with habitat connectivity. In some situations, this kind of models can be advantageous since their implementation does not require sophisticated mathematical tools while they can incorporate precise information on the species behaviour. However, they remain limited in terms of general theoretical analysis [12, 13, 14]. Conversely, diffusion models, although they sometimes make oversimplifying assumptions about movement of real organisms, provide an excellent tool for obtaining theoretical and qualitative results on populations dynamics, which can lead to a better understanding of some ecological processes. The idea of modelling population dynamics with these models from molecular physics has emerged at the beginning of the 20th century, with random walk theories of organisms, introduced by Pearson and Blackeman [15]. Then, Fisher [16] and Kolmogorov et al. [17] used a reaction-diffusion equation as a model for population genetics. Several years later, Skellam [18] used this type of model to study biological invasions, and he succeeded to propose quantitative explanations of observations, in particular of spreads of muskrats throughout Europe at the beginning of 20th Century. Since then, these models have been widely used to explain spatial propagation or spreading of biological species (bacteria, epidemiological agents, insects, fishes, plants, etc., see the books [19, 20, 21] for review).

The reaction-diffusion models that we study in this article correspond to 
a natural extension of the Fisher model to heterogeneous environments. This kind of model has been introduced by Shigesada et al. [22] and studied recently $[23,24,25,26]$, but it already appears that the heterogeneous character of the coefficients plays an essential role. Indeed, for different spatial configurations of the environment, the modelled species can tend to extinction or survive, disperse or not, depending on the arrangement of the habitat, as illustrated by the work of Roques and Stoica [27]. These models have been considered either in bounded domains $\Omega$ or in an infinite domain. This last case, $\Omega=\mathbb{R}^{N}$, is especially helpful when spreading phenomena are studied since it generates the existence of travelling waves [26, 28, 29, 30].

Cantrell and Cosner [31] have established, in the particular case of a 1dimensional bounded domain with reflecting boundary condition, that in a binary environment composed of habitat and non-habitat patches, it was better to concentrate all the habitat at a boundary of the domain. Shigesada and Kawasaki [24] proved a comparable result in the case of a 1-dimensional infinite domain, composed of periodic regions of habitat and non-habitat. They found that it was better for species persistence to concentrate the habitat patches at the centre of the period cell. Berestycki et al. [25] then generalised these results to the $\mathrm{N}$-dimensional case, in both bounded and infinite-periodic situations. To do so, they gave a necessary and sufficient condition for species survival, based on the instability of the state 0 (where no individuals are present). This condition is equivalent to the negativity of the smallest eigenvalue $\lambda_{1}$ of the linearised elliptic operator around the 0 state (such a criterion was also used in [31], in the bounded domain case). Using this criterion, they found a way of rearranging the habitat that decreases the value of $\lambda_{1}$. Thus, given an initial spatial configuration of the habitat, the rearranged and more aggregated configuration always provides better chances of species survival for the Shigesada et al. model, while it corresponds to the same habitat's area. When the space dimension $N$ is larger than 1, among these aggregated configurations, we do not know which one minimises $\lambda_{1}$.

In the 1-dimensional case, the question of the optimal shape of the habitat is already solved. As a result of the works [24, 25, 31], this shape is simply an interval. However, even in the 2-dimensional case, as far as we know, nothing is known about this optimal shape (even its existence is not established), except that, if it exists, the optimal shape has to be stable by the above-mentioned rearrangement process, as a consequence of the results in [25]. Mathematically, the problem is the same as minimising the eigenvalue $\lambda_{1}$ with respect to a coefficient of the equation, corresponding to habitat configuration. Thus it is a part of the vast area of eigenvalue minimisation problems, among which the most celebrated is surely the Rayleigh problem [32] of minimising the lowest frequency of a membrane. It says that, in the simple homogeneous Fisher's case, in bounded domain with lethal boundary and fixed area $A$, the principal eigenvalue $\lambda_{1}$ of the Dirichlet Laplacian is minimal when $\Omega$ is a ball. Thus if the modelled species survives in a domain of area $A$, then it automatically survives in a ball-shaped domain of area $A$, while in other domains of area $A$, the species may go to extinction. This result is a consequence of the classical 
Rayleigh-Faber-Krahn inequality [33, 34, 35, 36].

In this paper, we address the question of finding an optimal habitat configuration in bounded environments with reflecting (also called "Neumann") boundary conditions and in infinite-periodic environments. We prove the existence of an optimal configuration. Then, in the 2-dimensional case, we approximate it thanks to new numerical computations, which exhibit two thresholds in the habitat abundance. Between these two thresholds, the optimal configuration has the shape of a "stripe". These numerical results lead to new questions: (i) is there a relation between the shapes of the optimal configurations in the bounded and periodic cases? (ii) does the optimal configuration depend on the equation coefficients amplitude between the habitat and non-habitat regions? (iii) in the particular situation of an environment composed of stripes, are large habitat stripes separated with large gaps better than thin stripes with thin gaps? We solve these questions analytically, in any space dimension; however, for the sake of clarity, most of our statements are presented in the 2-dimensional case.

\section{Model formulation and preliminary mathemat- ical results}

\subsection{Formulation of the model}

The classical reaction-diffusion model in homogeneous environments of Fisher and Kolmogorov et al. corresponds to the following equation,

$$
\frac{\partial u}{\partial t}=D \nabla^{2} u+u(\mu-\nu u) \text { in } \Omega \subseteq \mathbb{R}^{N} .
$$

The unknown $u=u(t, x)$ corresponds to the population density at time $t$ and position $x=\left(x_{1}, \ldots, x_{N}\right)$. The number $D>0$ measures the dispersion rate and $\nabla^{2}$ stands for the spatial dispersion operator $\nabla^{2} u=\sum_{i=1}^{N} \frac{\partial^{2} u}{\partial x_{i}^{2}}, N$ being the spatial dimension. The constant real numbers $\mu>0$ and $\nu$ respectively correspond to the intrinsic growth rate of the modelled species and to its susceptibility to crowding.

The extension of this model to heterogeneous environments that we study in this paper (Shigesada et al. model) can be written as follows,

$$
\frac{\partial u}{\partial t}=D \nabla^{2} u+u(\mu(x)-\nu(x) u), \quad \text { in } \Omega \subseteq \mathbb{R}^{N} .
$$

We assume that $\mu(x)$ takes two values $\mu^{+}$or $\mu^{-}$, depending on $x$, with $\mu^{+}>\mu^{-}$. Regions where $\mu(x)=\mu^{+}$correspond to "habitat" and are favourable zones, whereas regions where $\mu(x)=\mu^{-}$are less favourable for the species ("nonhabitat" regions). When $\mu^{-}<0$, it means that the birth rate is less than the mortality rate in the non-habitat region. In this case, as proved in [25], the species may tend to extinction.

The function $\nu$ is bounded, $\nu \geq 0$ in $\Omega$ and $\nu$ is not equal to 0 almost everywhere, which corresponds to the classical negative density-dependence. 
We consider two kinds of domains: the bounded case, where $\Omega$ is a piecewise $C^{1}$ bounded domain; and the periodic case, with $\Omega=\mathbb{R}^{N}$ and the coefficients $\mu(x)$ and $\nu(x)$ are periodic in the sense that there exist $L_{1}, \ldots, L_{N}>0$ such that $\mu\left(x_{1}+k_{1} L_{1}, \ldots, x_{N}+k_{N} L_{N}\right)=\mu\left(x_{1}, \ldots, x_{N}\right)$ and $\nu\left(x_{1}+k_{1} L_{1}, \ldots, x_{N}+\right.$ $\left.k_{N} L_{N}\right)=\nu\left(x_{1}, \ldots, x_{N}\right)$ for all integers $k_{1}, \ldots, k_{N} \in \mathbb{Z}$ and for all $\left(x_{1}, \ldots, x_{N}\right)$ in $\mathbb{R}^{N}$. We denote $L=\left(L_{1}, \ldots, L_{N}\right)$ and we say that $\mu$ and $\nu$ are $L$-periodic. The period cell $C$ is defined by

$$
C:=\left[0, L_{1} \mid \times \cdots \times\left[0, L_{N}\right]\right.
$$

In the bounded case, we assume that we have Neumann boundary conditions: $\frac{\partial u}{\partial n}=0$ a.e. on $\partial \Omega$, where $n$ is the outward unit normal to $\partial \Omega$.

Note that the study in $\mathbb{R}^{N}$ with periodic coefficients cannot reduce to a study on a torus since the solution $u$ of $(2.2)$ is not periodic as soon as the initial condition $u(0, x)$ is not periodic.

In the periodic case, $C$ is defined by (2.3). In the bounded case, we set $C:=\Omega$. Moreover, in both cases, we assume that

$$
\text { there exist two Borel sets } C^{+}, C^{-} \subset C \text {, s.t. }\left\{\begin{array}{l}
\mu(x)=\mu^{+} \text {if } x \in C^{+}, \\
\mu(x)=\mu^{-} \text {if } x \in C^{-}, \\
C=C^{+} \cup C^{-},
\end{array}\right.
$$

so that $C^{+}$corresponds to the habitat regions and $C^{-}$to the non-habitat regions.

Definition 2.1 Let $\mu$ be a function satisfying (2.4). We say that $C^{+}(\mu):=C^{+}$ is the habitat configuration associated to the growth rate function $\mu$.

\subsection{A criterion for species persistence}

The necessary and sufficient condition of species survival that we use in this work is based on the sign of an eigenvalue $\lambda_{1}$.

This number $\lambda_{1}$ is the principal eigenvalue of the linear operator $-D \nabla^{2}-$ $\mu(x)$ and can be defined as the unique real number such that there exists a function $\Phi>0$ which satisfies

$$
\mathcal{L}_{0} \Phi:=-D \nabla^{2} \Phi-\mu(x) \Phi=\lambda_{1} \Phi \text { in } \Omega, \quad \Phi>0 \text { in } \Omega,
$$

either with $L$-periodicity in the periodic case, or with the boundary condition $\frac{\partial \Phi}{\partial n}=0$ a.e. on $\partial \Omega$ in the bounded case ( $\Phi$ is also unique up to multiplication by positive numbers, see Amann [37] for further details). As we can see on formula (2.5), $\lambda_{1}$ does not depend on the coefficient $\nu$. When necessary, we shall distinguish the principal eigenvalues in the periodic and bounded cases by denoting them $\lambda_{1, p}$ and $\lambda_{1, b}$ respectively. Furthermore, in order to emphasise the dependency of $\lambda_{1}$ with respect to $\mu$, we shall sometimes write $\lambda_{1}(\mu)$ for $\lambda_{1}$. Note that, given a function $\mu$ satisfying (2.4), the set $C^{+}$is uniquely defined. 
Reciprocally, the "patch function" $\mu$ is uniquely defined by $C^{+}, \mu^{+}$and $\mu^{-}$, and, in the periodic case, the periodicity conditions. Thus, for fixed values of $\mu+$ and $\mu^{-}$, we note

$$
\lambda_{1}\left[C^{+}\right]:=\lambda_{1}(\mu) .
$$

Since the operator $\mathcal{L}_{0}$ is self-adjoint, it is well-known that $\lambda_{1}$ satisfies the following formula:

$$
\lambda_{1}(\mu)=\min _{\varphi \in G \backslash\{0\}} \frac{\int_{C} D|\nabla \varphi|^{2}-\mu(x) \varphi^{2}}{\int_{C} \varphi^{2}},
$$

where $G$ is defined by $G:=H_{p e r}^{1}=\left\{\varphi \in H_{l o c}^{1}\left(\mathbb{R}^{N}\right)\right.$ such that $\varphi$ is L-periodic $\}$ in the periodic case and $G:=H^{1}(C)$ in the bounded case.

Given a bounded continuous nonnegative initial datum $u(0, x)=u_{0}(x)$, the behaviour of the solution $u(t, x)$ of $(2.2)$ in the periodic case is given in [25], Theorem 2.6. Namely, if $\lambda_{1} \geq 0$, then $u(t, x) \rightarrow 0$ uniformly in $\mathbb{R}^{N}$, thus the species tends to extinction, whereas if $\lambda_{1}<0, u(t, x) \rightarrow p(x)$ (uniformly on all compacts sets of $\mathbb{R}^{N}$ ), where $p(x)$ is the unique positive and bounded stationary solution of (2.2) i.e. $p$ satisfies

$$
D \nabla^{2} p(x)+p(x)(\mu(x)-\nu(x) p(x))=0, x \in \mathbb{R}^{N} .
$$

The existence, uniqueness and periodicity of $p$ are proved in [25], Theorems 2.1 and 2.4 .

In the bounded case, a similar result holds. Its proof, which is more classical, can be viewed as a straightforward adaptation of the results in [25] (see also Berestycki [38] for an analysis in the case of Dirichlet boundary conditions). Moreover, in the bounded case, the convergence to the stationary state, $u(t, x) \rightarrow p(x)$ as $t \rightarrow \infty$, is uniform in $\Omega$.

\subsection{Mathematical formulation of the optimisation prob- lem}

The habitat proportion $h \in[0,1]$ being fixed, we define the family of habitat configurations with proportion $h$ by:

$$
S_{h}:=\left\{\text { Borel sets } C^{+} \subset C \text {, such that }\left|C^{+}\right|=h|C|\right\},
$$

where $|E|$ denotes the Lebesgue measure of a set $E$.

This habitat proportion $h$ and the values $\mu^{+}$and $\mu^{-}$of the growth rate in the habitat and nonhabitat regions being fixed, our goal is to describe the optimal habitat configurations for species survival, that is, the sets $C^{+} \in S_{h}$ which minimise $\lambda_{1}\left[C^{+}\right]$. For given values of $\mu^{+}$and $\mu^{-}$, we denote the optimal value of $\lambda_{1}\left[C^{+}\right]$by

$$
\underline{\lambda_{1}}:=\min _{C^{+} \in S_{h}} \lambda_{1}\left[C^{+}\right],
$$


and by $\underline{S}_{h, \mu^{+}, \mu^{-}}$the set of optimal habitat configurations:

$$
\underline{S}_{h, \mu^{+}, \mu^{-}}:=\left\{C^{+} \in S_{h}, \text { such that } \lambda_{1}\left[C^{+}\right]=\underline{\lambda_{1}}\right\} .
$$

Remark 2.2 For a fixed $h$ value, $\underline{\lambda_{1}}$ depends on $\mu^{+}$and $\mu^{-}$. Moreover, as we will see in the next sections, the set $\underline{S}_{h, \mu^{+}, \mu^{-}}$may also depend on $\mu^{+}$and $\mu^{-}$ as soon as $N \geq 2$.

The existence of such optimal configurations is given by:

Theorem 2.3 For all $\mu^{+}>\mu^{-}$in $\mathbb{R}$, and for all $h \in[0,1]$, the set $\underline{S}_{h, \mu^{+}, \mu^{-}}$is not empty.

Proof. This result is proved rigourously in Appendix A.

When needed, we may distinguish in the sequel the sets of optimal habitat configurations in the bounded and periodic cases, denoting them by $\underline{S}_{h, \mu^{+}, \mu^{-}}$ and $\underline{S}_{\underline{p}_{, \mu^{+}, \mu^{-}}}$, respectively.

Remark 2.4 Actually, the minimum of $\lambda_{1}(\mu)$ among the functions $\mu$ in $\{\mu \in$ $L^{\infty}(C), \mu^{-} \leq \mu \leq \mu^{+}$a.e. and $\left.\frac{1}{|C|} \int_{C} \mu=h \mu^{+}+(1-h) \mu^{-}\right\}$is attained on a "patch function" satisfying (2.4). This result, which says that the optimal configuration is of "bang-bang" type, is proved in Nadin [39].

\subsection{A numerical method for finding an optimal habitat configuration in the bounded case}

We consider here the two-dimensional bounded case with $C=\left[0, L_{1}\right] \times\left[0, L_{2}\right]$. The domain is covered by $n$ disjoint rectangles, and $C^{+}$is the union of $n^{+}$of these rectangles (see Fig. 1). Moreover, we set $n=n_{r} \times n_{c}$ where $n_{r}$ is the number of rows and $n_{c}$ is the number of columns in $C$. We have numerically computed the value of $\lambda_{1}$ for different configurations of $C^{+}$and $C^{-}$. Among the huge number of possible configurations $\left(\mathrm{C}_{n}^{n^{+}}=n ! /\left(n^{+} !\left(n-n^{+}\right) !\right)\right)$, the optimal ones can be sought in a smaller subset of admissible configurations. Indeed, consider the configuration $C_{n_{r}, n_{c}}^{+, *}$ obtained with the following algorithm: 1 . on each row, the rectangles that belong to $C^{+}$are moved to the left; 2 . then, on each column the rectangles that belong to $C^{+}$are moved down. The old and new habitat configurations $C^{+}$and $C_{n_{r}, n_{c}}^{+, *}$ have the same area and it follows from Theorem 6.3 in [25], that $\lambda_{1}\left[C^{+, *}\right] \stackrel{\leq}{\leq} \lambda_{1}\left[C^{+}\right]$. Thus, we only have to look for the optimal configuration among the habitat which are stable through this algorithm, that is, habitats concentrated in a corner of the domain. The number of configurations $N_{\text {conf }}$ corresponds to the number of ways of writing the integer $n^{+}$as a sum of $n_{c}$ integers, each one being smaller than or equal to $n_{r}$. It can be computed in a similar way as the "partition function" (see e.g. [40]). For our computations, we fixed $C=[0,1] \times[0,1], \mu^{+}=10, \mu^{-}=-1, n_{r}=n_{c}=15$, $n=15 \times 15=225$, and $n^{+}$varies between 0 and 225 , to investigate all the levels of habitat proportion. The number $N_{\text {conf }}$ varies between $1\left(n^{+}=0,1,224,225\right)$ to $2,527,074$ (for $n^{+}=112,113$; compare with $C_{225}^{112} \simeq 2.8 \cdot 10^{66}$ ). 


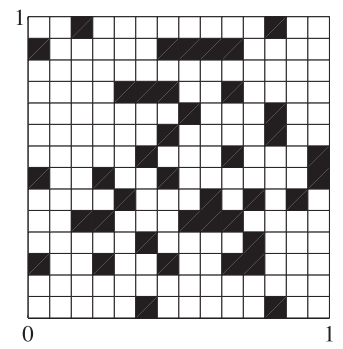

Figure 1: An example of a configuration $C^{+} \in S_{h}$, with $h=0.2$, in the particular case of a domain $C=[0,1]^{2}$, covered by $n=225$ disjoint rectangles, and where $C^{+}$is the union of $n^{+}=45$ of these rectangles. The habitats regions are depicted in black.

As the mesh is refined i.e. when $n_{r}$ and $n_{c}$ increase, the "discrete" optimal habitat configurations $C_{n_{r}, n_{c}}^{+, *}$, obtained by the above process, converge to an optimal configuration $C^{+, *} \in \underline{S}_{h=\frac{n^{+}}{n}, \mu^{+}, \mu^{-}}$. Indeed, let us define the distance between two subsets $A_{1}$ and $A_{2}$ of $C$ by the measure of the symmetric difference of $A_{1}$ and $A_{2}$,

$$
\mathrm{d}_{\text {set }}\left(A_{1}, A_{2}\right)=\left|\left(A_{1} \cup A_{2}\right)-\left(A_{1} \cap A_{2}\right)\right|,
$$

and the distance between a subset $A \subset C$ and the family of subsets $\underline{S}_{h, \mu^{+}, \mu^{-}}$by

$$
\mathrm{d}_{s e t}\left(A, \underline{S}_{h, \mu^{+}, \mu^{-}}\right)=\inf _{K \in \underline{S}_{h, \mu^{+}, \mu^{-}}} \mathrm{d}_{s e t}(A, K),
$$

then, we have the following

Proposition 2.5 As $n_{r}, n_{c} \rightarrow+\infty$, d $d_{s e t}\left(C_{n_{r}, n_{c}}^{+, *}, \underline{S}_{h, \mu^{+}, \mu^{-}}\right) \rightarrow 0$ as $n_{r}, n_{c} \rightarrow$ $+\infty$.

Proof. This result is proved in Appendix B.

\subsection{Deducing the periodic optimal configuration from the bounded case}

With a numerical method comparable to that of $\S 2.4$, using Theorem 2.11 in [25], we could compute an optimal habitat configuration in the periodic case. However, the computations would be lengthy whereas we can directly deduce it from the bounded case. Indeed,

Theorem 2.6 For fixed numbers $\mu^{+}, \mu^{-} \in \mathbb{R}$, with $\mu^{+}>\mu^{-}$, and $h \in[0,1]$, let $C_{b}^{+, *}$ be an optimal habitat configuration in $\underline{S}_{h, \mu^{+}, \mu^{-}}$in the bounded case. Assume that $C_{b}^{+, *}$ is stable by monotone rearrangement with respect to each variable, with $C=\left[0, L_{1} \mid \times\left[0, L_{2}\right]\right.$. Let $C_{p}^{+, *}$ be the set obtained by reflecting $C_{b}^{+, *}$ with respect to each axis:

$$
C_{p}^{+, *}:=C_{b}^{+, *} \cup R_{1}\left[C_{b}^{+, *}\right] \cup R_{2}\left[C_{b}^{+, *} \cup R_{1}\left[C_{b}^{+, *}\right]\right],
$$


where $R_{i}$ is the reflection with respect to the axis $\left\{x_{i}=L_{i}\right\}$, for $i=1,2$. Then $C_{p}^{+, *}$ is an optimal periodic configuration in $\underline{S}_{p_{h, \mu^{+}, \mu^{-}}}$on $\tilde{C}=\left[0,2 L_{1} \mid \times\left[0,2 L_{2}\right]\right.$.

Proof. In the bounded case, the existence of an optimal configuration, stable by monotone rearrangement with respect to each variable, follows from the proof of Theorem 2.3. The remaining part of the proof is given in Appendix C. We have proved a comparable result in any space dimension $N$.

Thus, using our numerical computations in the bounded case, and from Proposition 2.5, we can derive an approximation of the optimal configuration in the periodic case.

\section{Results}

Both numerical and analytical results are presented in this section, in the bounded and periodic cases.

\subsection{The bounded case}

Using the numerical method presented in $\S 2.4$, we obtained that, when the proportion of habitat $h=\frac{n^{+}}{n}$ is small, the optimal habitat regions have the shape of a quarter of disc (Fig. 2). When this proportion is close to 1 , these optimal configurations look like the complement of a quarter of disc. When $h$ is not too small nor too large, the optimal configurations are stripe-shaped, and are aggregated along a side of the boundary of the domain.

In the particular case of striped configurations, we have proved the following result:

Theorem 3.1 Let $\mu^{+}>\mu^{-}$, and $h \in[0,1]$. Assume that $L_{1} \neq L_{2}$, and let $S_{h}^{s}$ be the subset of $S_{h}$ made of striped configurations. Then $\min _{C^{+} \in S_{h}^{s}} \lambda_{1}\left[C^{+}\right]$is attained for the striped configuration concentrated along the smallest side of the domain.

Proof. See Appendix D

This result is illustrated on Fig. 3.

When the habitat shape $C^{+}$is fixed a priori as a ball, it is proved in [41] that the optimal position of the ball has to be tangent to the boundary of the domain, as depicted in Fig. 4. However, this configuration is never optimal among all habitats of fixed area when the shape of the habitat is not fixed a priori:

Proposition 3.2 Let $\mu^{+}>\mu^{-}$, and $h \in[0,1]$. Assume that $C^{+} \in S_{h}$ is tangent to a boundary of the domain, in the sense that there exist a point $x_{0} \in \partial C$ and a ball $B \subset C^{+}$such that $x_{0} \in \partial B \cap \partial\left(C \backslash C^{+}\right)^{\circ}$. Then $\lambda_{1}\left[C^{+}\right]>\underline{\lambda_{1}}$. Equivalently, this means that $C^{+} \notin \underline{S}_{h, \mu^{+}, \mu^{-}}$. 


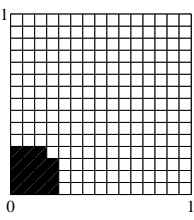

(a) $n^{+}=15$

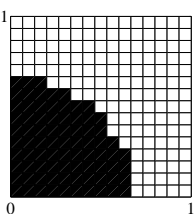

(d) $n^{+}=80$

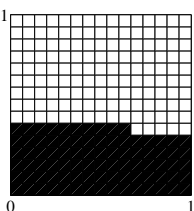

(g) $n^{+}=85$

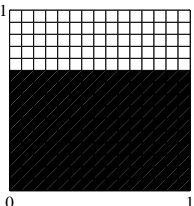

(j) $n^{+}=150$

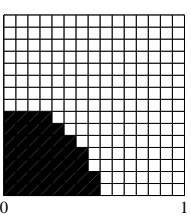

(b) $n^{+}=45$

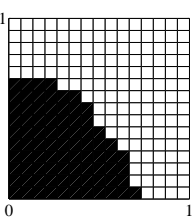

(e) $n^{+}=82$

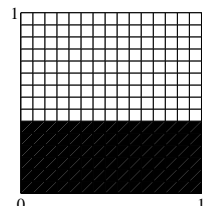

(h) $n^{+}=90$

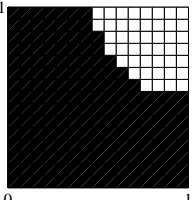

(k) $n^{+}=180$

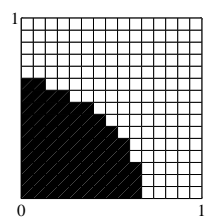

(c) $n^{+}=75$

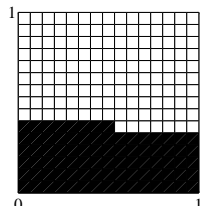

(f) $n^{+}=83$

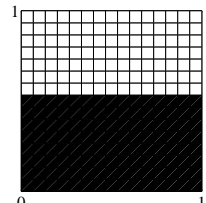

(i) $n^{+}=120$

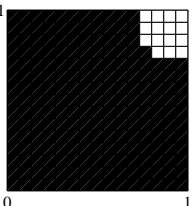

(l) $n^{+}=210$

Figure 2: The optimal habitat configuration in terms of the number $n^{+}$of habitat cells in the case of a bounded environment with Neumann boundary conditions. The black cells correspond to habitat and the white cells to nonhabitat. 


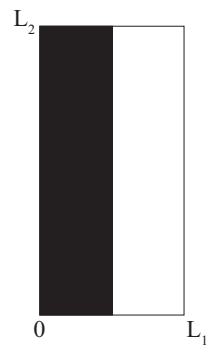

(a)

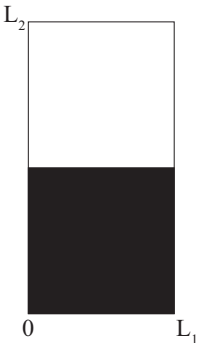

(b)

Figure 3: Two striped configurations. The habitat regions are depicted in black. In the bounded and periodic cases, the configuration (b), where the habitat $C^{+}$ is aggregated along the smallest side of the domain provides lower values of $\lambda_{1}$ than the configuration (a), independently of the habitat proportion $h$, and of $\mu^{+}$and $\mu^{-}$.

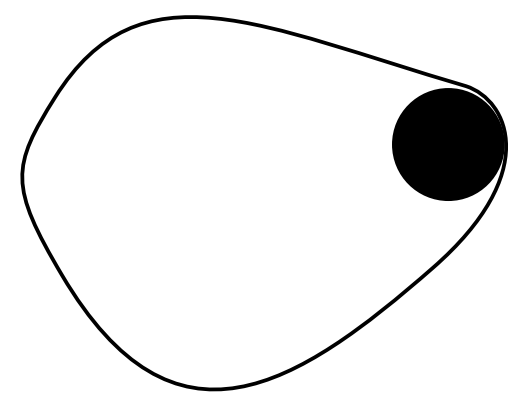

Figure 4: A configuration which is not optimal in the bounded case. The habitats regions $C^{+}$are depicted in black.

Proof. This is a consequence of Steps 2 and 3 of Appendix E. See Remark 4.1.

\subsection{The periodic case}

As in the bounded case, when the proportion of habitat is small, the optimal configuration of $C^{+}$is disc-shaped (Fig. 5). Then, for a larger proportion, it takes the form of a stripe, and when the proportion of habitat approaches 1 , it looks like the complement of a disc. When the habitat region is stripeshaped, and when the period cell is not a square, two types of configurations exist. Similarly to Theorem 3.1, we formally prove in Appendix D that the configuration with wide stripes of habitat and wide gaps is always better than the configuration with narrow stripes and narrow gaps between the stripes.

These computations show that the shape of the optimal habitat configu- 


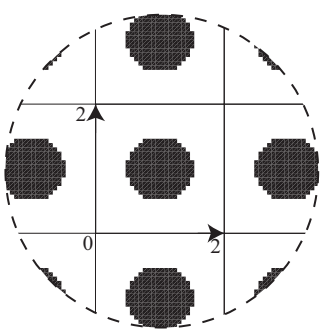

(a) $h=0.2$

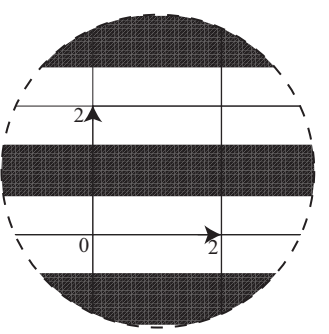

(b) $h=0.4$

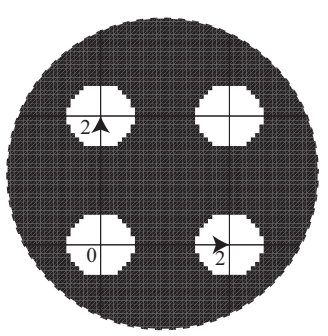

(d) $h=0.8$

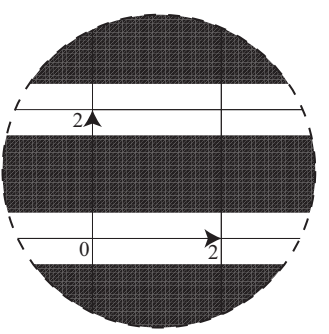

(c) $h=0.6$

Figure 5: Approximation of an optimal habitat configuration in the periodic case, with a period cell of size $2 \times 2$, in terms of the habitat abundance $h=$ $\left|C^{+}\right| /|C|$, with $\mu^{-}=-1, \mu^{+}=10$. The black regions correspond to habitat and the white regions to non-habitat. The figures are assumed to extend in all direction indefinitely. 
ration is highly dependent on the proportion of habitat, even if two types of optimal shapes (disc and stripe) are numerically observed. This result rules out the attractive conjecture of the optimality of the striped configurations for all habitat abundance. Actually, we can prove formally the following result:

Theorem 3.3 Assume that $N=2$. Let $\mu^{+}>\mu^{-}$be fixed.

(a) The disc configuration is not always optimal: assume that $h=\frac{\pi\left(\min \left\{L_{1}, L_{2}\right\}\right)^{2}}{4|C|}$, and that $C^{+}$is a disc in $S_{h}$. Then $\lambda_{1}\left[C^{+}\right]>\underline{\lambda_{1}}$.

(b) The stripe configuration is not always optimal: there exist $L_{1}, L_{2}>0$ and $h \in[0,1]$ such that for all stripe $C^{+}$in $S_{h}, \lambda_{1}\left[C^{+}\right]>\underline{\lambda_{1}}$.

Proof. Part (a) follows from Appendix E, Steps 2 and 3, and Part (b) follows from Appendix F. We proved similar results in any space dimension $N \geq 2$.

From Theorem 2.6, it follows that these results are also true in the bounded case, with, for Part (a), a quarter of disc instead of a disc.

\subsection{Dependence of the optimal shape with respect to the difference between the growing rates $\mu^{+}$and $\mu^{-}$}

Let the proportion of habitat $h$ be fixed. It then turns out that the optimal habitat configuration does not depend directly on the values of $\mu^{+}$and $\mu^{-}$, but only on the difference $b:=\mu^{+}-\mu^{-}$. Indeed, as a consequence of formula (2.6), for all constant $k \in \mathbb{R}$, we obtain

$$
\lambda_{1}(\mu-k)=\lambda_{1}(\mu)+k .
$$

Thus, we may note $\underline{S}_{h, b}$ the set of optimal habitat configurations, with $b=$ $\mu^{+}-\mu^{-}$, instead of $\underline{S}_{h, \mu^{+}, \mu^{-}}$.

In dimension 1 , when $C:=\left[0, L_{1}\right]$, the optimal habitat shape is in fact totally independent of $\mu^{+}$and $\mu^{-}$. Indeed, in the periodic case, and up to a shift of the period cell, there is an unique configuration where $C^{+}$is connected, which is therefore the optimal one (see [25]). A similar result holds in the bounded case, where the optimal habitat configuration is an interval situated at either end of $\left[0, L_{1}\right]$.

However, in dimension 2 or higher, given the proportion of habitat $h$, we prove that the optimal shape of the habitat does truly depend on the amplitude $b$ of the growth rate $\mu$ :

Theorem 3.4 Assume that $N \geq 2$. There exists $h \in[0,1]$ such that, for all $C^{+} \in S_{h}$, there exists $b>0$ such that $C^{+} \notin \underline{S}_{h, b}$.

Proof. This is proved in Appendix E. The proof relies on the fact that (i) when the amplitude tends to infinity, the optimal habitat in the periodic case tends to aggregate in a ball; (ii) from Theorem 3.3, Part (a), the ball-shaped configuration of Fig. 6 is never optimal whatever the positive number $b$ is.

This result of dependence with respect to amplitude is also true in the bounded case. Nevertheless, the transition between disc-shaped and stripe- 


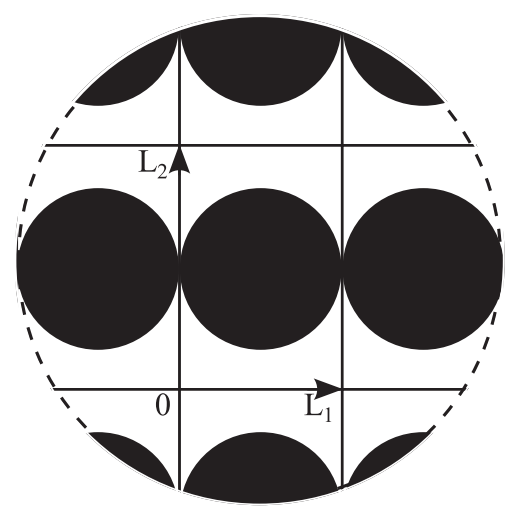

Figure 6: A periodic configuration which is not optimal. The habitats regions are depicted in black.

shaped configurations as the proportion of habitat increases seems to hold for a wide range of values of $b$, as observed numerically in dimension 2. As the amplitude $b$ increases, the ball configuration becomes better than the striped configuration for a wider range of habitat proportion (Fig. 7).

\section{Discussion}

We have numerically derived the optimal configurations of the habitat in the case of a 2-dimensional patchy environment, constituted of cells of habitat and non-habitat regions, distributed on a grid. As the size of the elementary cells decrease, these numerical configurations converge to an optimal habitat configuration for fixed values of habitat abundance and growth rates. This configuration is a global minimiser of $\lambda_{1}$, contrary to most of the numerical methods for minimising eigenvalues under geometrical constraints (see e.g. [42, 43]). The shape of these optimal configurations was not known before for dimensions higher than 1.

In rectangular bounded domains of $\mathbb{R}^{2}$, with reflecting boundaries, we have observed a transition effect from "quarter of disc" to "striped" and to "complement of a quarter of disc" optimal configurations as the proportion of habitat increases. The same phenomenon occurs in infinite periodic environments, with discs instead of quarter of discs. The transition occurs abruptly, and the striped configurations remain optimal for a substantial range of habitat proportion.

Actually, we have proved that the optimal configurations in the bounded and periodic cases can be deduced from each other. This means that, in a period cell, the role played by the proximity of the habitat patches lying in the other period cells is similar to the boundary effects of the bounded case.

From Theorem 6.3 in [25], and as recalled in the introduction of this paper, $\lambda_{1}$ decreases, and thus the chances of survival increase, when the habitat is 


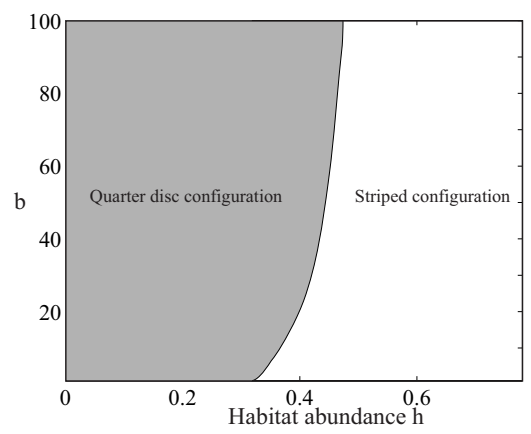

Figure 7: Comparison between the quarter disc configuration and the striped configuration, in the bounded case of a square $C=[0,1] \times[0,1]$, in terms of the amplitude of the difference between the growth rates $b$, and of the habitat abundance $h=\left|C^{+}\right| /|C|$. In the shadowed area, the quarter disc configuration provides lower values of $\lambda_{1}$, while in white zone the striped configuration gives lower $\lambda_{1}$ values.

rearranged in a monotone way, with respect to each space variable. Thus, the optimal configurations are always aggregated against the domain boundary. This underlines the favourability of the boundary effects. From an animal point of view, these effects can be interpreted as follows: the individuals situated in the habitat which try to cross the boundary are rejected into the habitat; therefore, the average time spent in the habitat region by each individual is larger when the habitat is closer to a boundary. From a vegetal viewpoint less descendants disperse in the unfavourable regions. Conversely, the edge of the habitat seems to have detrimental effects. This can again be illustrated with Theorem 6.3 in [25]. Indeed, in the rearranged configurations the habitat has a small perimeter compared to situations where the habitat is more "fragmented".

Hence, the optimal configuration is a compromise between lessening the detrimental habitat edge effects, which means that the perimeter of the habitat has to be minimal and leads to disc shapes, and taking advantage of the domain boundary (or the proximity of other patches in the periodic case), leading to striped shapes. However, both phenomena cannot be optimised simultaneously. When the habitat abundance is low, the optimal configuration looks like a quarter of disc in the bounded case, and looks like a disc in the periodic case; in these situation, the edge of the habitat is reduced, but the population does not take full advantage of the domain boundary. When the habitat abundance increases, the optimal configuration becomes stripe-shaped, and is concentrated along a side of the boundary of the domain. Thus, in that case, the positive influence of the boundary effects is important, compared to the detrimental habitat edge effects. However, when the domain (or period cell in the periodic case) is not a square, we prove formally that the best striped habitat configuration is aggregated along the smallest side of the domain. Thus this configuration 
reduces the edge in contact with non-habitat regions, while it does not take full advantage of the boundary, still realising a compromise between these edge and boundary effects. In the periodic case, this means that the configuration with wide stripes is always better than the one with narrow stripes. This gives an element of explanation of the observation of Kinezaki et al. [30] that, in a periodic environment composed of stripes, when the scale of fragmentation is enlarged without changing the habitat proportion, the spreading speed of the modelled species increases.

We have proved that this equilibrium between boundary and edge effects also depends on amplitude of the difference between the growth rates in the habitat and non-habitat regions. As this amplitude grows, the detrimental effects of the habitat edge become more important compared to the favourable boundary effects, resulting in ball-shaped optimal configurations in highly contrasted environments. Nevertheless, for a wide range of values of the growth rate function, we observed the same transition behaviour between disc-shaped and striped configurations as the habitat abundance increases.

Our results in the bounded case largely depend on the reflecting (Neumann) boundary conditions. These boundary conditions mean that individuals encountering the boundary are reflected inside the domain. They can be encountered in many real-world situations, at different scales, corresponding, for instance, to the walls of a laboratory arena for small terrestrial species (see e.g. the experiment of Hannunen and Ekbom [44]), to fences or rivers surrounding gardens, forests or natural parks $[24,45]$ for larger species. This type of boundary conditions can also correspond to coasts, in the case of aquatic species. They can also be used for modelling plant dispersal, particularly when the seeds are dispersed by animals. Of course, other boundary conditions could have been envisaged. Of particular practical interest are also the absorbing (Dirichlet) conditions, that we briefly discussed in the introduction section. These conditions, where the domain's boundary "absorbs" all individuals encountering it, are often envisaged for species with passive movement, such as plants. Although most of the methods developed in this paper still work under these conditions, we have not considered here the issue of finding an optimal habitat configuration in this case. Firstly, because some numerical and theoretical results already exist in the mathematical literature [42]. Moreover, the question of optimal configuration should be of less interest in this case. Indeed, due to the negative effect of the domain's boundary, the habitat will concentrate at the centre of the domain. Therefore we cannot expect great changes in the topology of the optimal habitat configuration with respect to the model parameters.

A natural question arises: what is the interest of minimising $\lambda_{1}$ as soon as $\lambda_{1}<0$, since species survival is already granted? The answer is partially given by (3.12): $\lambda_{1}(\mu-k)=\lambda_{1}(\mu)+k$ for all $k \in \mathbb{R}$. Thus, a species in an environment with smaller values of $\lambda_{1}$ will be more robust to perturbations in its habitat. Moreover, as shown by [46] it is also more robust to external perturbations corresponding for instance to harvesting, in the sense that $\delta$ can be chosen higher in $\frac{\partial u}{\partial t}=D \nabla^{2} u+u(\mu(x)-\nu(x) u)-\delta$, without risking the 
species extinction.

Another natural question is: when the environmental heterogeneity also concerns the motility of the individuals, what results can be expected? Consider the equation

$$
u_{t}=\nabla^{2}(D(x) u)+u(\mu(x)-\nu(x) u),
$$

where the diffusion term $\nabla^{2}(D(x) u)$ is in the Fokker-Planck form. The coefficient $D(x)$ corresponds to the species motility (see e.g.[19, 21]). Setting $v(t, x):=D(x) u(t, x)$, and using some results of [25], we can obtain that the equation (4.13) exhibits the same behaviour as (2.2), replacing the initial growth term $\mu(x)$ by $\mu(x) / D(x)$. Thus, our above optimisation results also hold for equation (4.13), when $D(x)$ is rearranged along with $\mu(x)$, and in the same way. Such a rearrangement is biologically meaningful, since the motility and the growth terms are generally associated with a certain type of habitat, and are therefore spatially correlated. Note that, for most species that engage in active habitat selection, such as birds, mammals of amphibians, $D(x)$ is all the smaller as $\mu(x)$ is large, since they tend to slow down as they encounter favourable areas (e.g. [44]). Thus the functions $\mu$ and $\mu / D$ vary in the same way.

\section{Acknowledgements}

The authors are grateful to Etienne K. Klein for his valuable comments and suggestions.

\section{Appendices}

This section is devoted to the proofs of the mathematical results which were stated throughout this paper. The results of the appendices A,B,D,E,F are true in both periodic case and bounded case when $\Omega$ is a rectangle. However, we decided, for the sake of simplicity, and since the proof in one case can easily be adapted to the other, to prove each result in only one case.

\section{A. Existence of an optimal configuration}

The existence of at least one optimal configuration that maximises the chances of species persistence is proved in the periodic case. For another proof, in the Dirichlet case, see [42].

Let $\mu^{-}<\mu^{+}$be two given real numbers, $L_{1}, \ldots, L_{N}$ be $N$ given positive real numbers, and $h \in[0,1]$. Call $L=\left(L_{1}, \ldots, L_{N}\right)$ and

$$
C:=\left[-\frac{L_{1}}{2}, \frac{L_{1}}{2}\right] \times \cdots \times\left[-\frac{L_{N}}{2}, \frac{L_{N}}{2}\right] .
$$


For the sake of simplicity of the following calculations, the cell $C$ has been centred at the origin. Let $P_{h}$ be the set of measurable functions defined by:

$$
P_{h}:=\left\{\mu \text { satisfying (2.4) with }\left|C^{+}\right|=h|C|\right\},
$$

and

$$
\underline{\lambda_{1}}:=\inf _{\mu \in P_{h}} \lambda_{1}(\mu)=\inf _{C^{+} \in S_{h}} \lambda_{1}\left[C^{+}\right],
$$

where $\lambda_{1}(\mu)$ denotes the principal eigenvalue of the operator $-D \nabla^{2}-\mu$ with $L$-periodicity in the variables $x_{1}, \ldots, x_{N}$ (each function $\mu$ in $P_{h}$ can be extended by $L$-periodicity). By definition of $\lambda_{1}$, there exists a sequence $\left(\mu_{n}\right)_{n \in \mathbb{N}}$ in $P_{h}$, such that $\lambda_{1}\left(\mu_{n}\right) \rightarrow \underline{\lambda_{1}}$ as $n \rightarrow+\infty$. Since, for all $n \in \mathbb{N}, \lambda_{1}\left(\mu_{n}^{*}\right) \leq \lambda_{1}\left(\mu_{n}\right)$ and $\mu_{n}^{*} \in P_{h}$, we also have

$$
\lambda_{1}\left(\mu_{n}^{*}\right) \rightarrow \underline{\lambda_{1}} \text { as } n \rightarrow+\infty,
$$

where $\mu_{n}^{*}$ is the symmetric decreasing Steiner rearrangement of $\mu_{n}$ with respect to each variable $x_{1}, \ldots, x_{N}$ in the cell $C$ (see [25] for details). Up to a change on a set of zero measure, the functions $\mu_{n}^{*}$ can be constructed so that, for each $1 \leq i \leq$ $N$ and $\left(x_{1}, \ldots, x_{i-1}, x_{i+1}, \ldots, x_{N}\right) \in\left[-L_{1} / 2, L_{1} / 2\right] \times \cdots \times\left[-L_{i-1} / 2, L_{i-1} / 2\right] \times$ $\left[-L_{i+1} / 2, L_{i+1} / 2\right] \times \cdots \times\left[-L_{N} / 2, L_{N} / 2\right]$, there exists $a \in\left[0, L_{i} / 2\right]$ such that the function $s \mapsto \mu_{n}^{*}\left(x_{1}, \ldots, x_{i-1}, s, x_{i+1}, \ldots, x_{N}\right)$ is equal to $\mu^{+}$in $[-a, a]$ and to $\mu^{-}$in $\left[-L_{i} / 2, L_{i} / 2\right] \backslash[-a, a]$.

Up to the extraction of some subsequence, $\mu_{n}^{*}(x) \rightarrow \mu^{*}(x) \in\left\{\mu^{-}, \mu^{+}\right\}$as $n \rightarrow+\infty$, for all $x$ in the countable set $\mathbb{Q}^{N} \cap C$. Let $\Omega^{+}$be the closure of $\{x \in$ $\left.\mathbb{Q}^{N} \cap C, \mu^{*}(x)=\mu^{+}\right\}$. We extend the function $\mu^{*}$ to $C$ by setting $\mu^{*}(x)=\mu^{+}$ if $x \in \Omega^{+}$and $\mu^{*}(x)=\mu^{-}$otherwise; next, $\mu^{*}$ is extended by $L$-periodicity in $\mathbb{R}^{N}$. From the construction of the functions $\mu_{n}^{*}$, the function $\mu^{*}$ satisfies the same symmetry properties as $\mu_{n}^{*}$, in the sense that if $x=\left(x_{1}, \ldots, x_{N}\right) \in \Omega^{+}$, then $\mu^{*}(y)=\mu^{+}$for all $y \in\left[-\left|x_{1}\right|,\left|x_{1}\right|\right] \times \cdots \times\left[-\left|x_{N}\right|,\left|x_{N}\right|\right]$. Observe also that the boundary of $\Omega^{+}$has a zero $N$-dimensional Lebesgue measure.

Let $x=\left(x_{1}, \ldots, x_{N}\right)$ be an interior point of $C \backslash \Omega^{+}$. There exists $y=$ $\left(y_{1}, \ldots, y_{N}\right)$ another interior point in this set, such that $y \in \mathbb{Q}^{N}$ and

$$
\left|y_{i}\right|<\left|x_{i}\right| \text { for all } i=1, \ldots, N .
$$

Since $x, y \notin \Omega^{+}, \mu^{*}(x)=\mu^{*}(y)=\mu^{-}$. Moreover, by pointwise convergence, $\mu_{n}^{*}(y)=\mu^{-}$for $n$ large enough. From the symmetry properties of $\mu_{n}^{*}$, and from (4.15), we have $\mu_{n}^{*}(y) \geq \mu_{n}^{*}(x)$, therefore, $\mu_{n}^{*}(x)=\mu^{-}\left(=\mu^{*}(x)\right)$ for $n$ large enough. If now $x=\left(x_{1}, \ldots, x_{N}\right)$ is an interior point of $\Omega^{+}$, there exists another point $y$ in this set such that $y \in \mathbb{Q}^{N}$ and $\left|y_{i}\right|>\left|x_{i}\right|$ for all $i=$ $1, \ldots, N$, and we similarly obtain $\mu_{n}^{*}(x)=\mu^{+}\left(=\mu^{*}(x)\right)$ for $n$ large enough. The above observations imply that $\mu_{n}^{*}(x) \rightarrow \mu^{*}(x)$ as $n \rightarrow+\infty$ almost everywhere in $C$. Since the functions $\mu_{n}^{*}$ are uniformly bounded, Lebesgue's dominated convergence theorem yields $\mu_{n}^{*} \rightarrow \mu^{*}$ in $L^{1}(C)$ as $n \rightarrow+\infty$. Therefore, $\mu^{*} \in P_{h}$. 
Define, for all $\varphi \in H_{p e r}^{1} \backslash\{0\}$ and $\mu \in P_{h}$,

$$
Q_{\mu}(\varphi)=\frac{\int_{C} D|\nabla \varphi|^{2}-\mu(x) \varphi^{2}}{\int_{C} \varphi^{2}}
$$

Then,

$$
Q_{\mu^{*}}(\varphi) \leq Q_{\mu_{n}^{*}}(\varphi)+\frac{\int_{C}\left|\mu^{*}-\mu_{n}^{*}\right| \varphi^{2}}{\int_{C} \varphi^{2}} \text { for all } \varphi \in H_{p e r}^{1} \backslash\{0\} .
$$

Call $\Phi_{n}$ the principal eigenfunction associated to $\mu_{n}^{*}$, with the normalisation condition $\left\|\Phi_{n}\right\|_{\infty}=1$. That is, $\Phi_{n}$ is $L$-periodic, positive, and it satisfies

$$
-D \nabla^{2} \Phi_{n}-\mu_{n}^{*} \Phi_{n}=\lambda_{1}\left(\mu_{n}^{*}\right) \Phi_{n} \text { in } \mathbb{R}^{N} \text {. }
$$

By multiplying this equation by $\Phi_{n}$ and integrating over $C$, we get $Q_{\mu_{n}^{*}}\left(\Phi_{n}\right)=$ $\lambda_{1}\left(\mu_{n}^{*}\right)$. Hence,

$$
Q_{\mu^{*}}\left(\Phi_{n}\right) \leq \lambda_{1}\left(\mu_{n}^{*}\right)+\frac{\int_{C}\left|\mu^{*}-\mu_{n}^{*}\right|}{\int_{C} \Phi_{n}^{2}},
$$

and since $\lambda_{1}\left(\mu^{*}\right)=\min _{\varphi \in H_{p e r}^{1} \backslash\{0\}} Q_{\mu^{*}}(\varphi)$,

$$
\lambda_{1}\left(\mu^{*}\right) \leq \lambda_{1}\left(\mu_{n}^{*}\right)+\frac{\int_{C}\left|\mu^{*}-\mu_{n}^{*}\right|}{\int_{C} \Phi_{n}^{2}} .
$$

Moreover, since $\mu^{-} \leq \mu_{n}^{*} \leq \mu^{+}$in $\mathbb{R}^{N}$ and $-\mu^{+} \leq \lambda_{1}\left(\mu_{n}^{*}\right) \leq-\mu^{-}$, the normalisation condition $\left\|\Phi_{n}\right\|_{\infty}=1$ together with standard elliptic estimates (see Gilbarg and Trudinger [48]) imply that the sequence $\left(\Phi_{n}\right)_{n \in \mathbb{N}}$ is bounded in $C^{1}\left(\mathbb{R}^{N}\right)$ (at least), whence $\inf _{n \in \mathbb{N}}\left\|\Phi_{n}\right\|_{L^{2}(C)}>0$. Thus, passing to the limit as $n \rightarrow+\infty$ in (4.18), we get, since $\mu_{n}^{*} \rightarrow \mu^{*}$ in $L^{1}(C), \lambda_{1}\left(\mu^{*}\right) \leq \lambda_{1}$. It then follows from the definition of $\lambda_{1}$ that $\lambda_{1}\left(\mu^{*}\right)=\lambda_{1}$. Since $\mu^{*} \in P_{h}$, the associated habitat configuration $C^{+, *}$, defined by $(2.4)$, belongs to $S_{h}$, and $\lambda_{1}\left[C^{+, *}\right]=\lambda_{1}$, therefore $C^{+, *}$ is an optimal configuration. The infimum in the definition of $\underline{\lambda_{1}}$ is then a minimum.

\section{B. Convergence of the computed configurations to optimal configurations as the mesh is refined}

This time, the proof is done in the bounded case of a rectangle. We assume that

$$
C:=\left[0, L_{1}\right] \times \cdots \times\left[0, L_{N}\right] .
$$


Let $\mu^{+}, \mu^{-} \in \mathbb{R}$, and fix the habitat proportion $h=\frac{n^{+}}{n}$. Let $P_{h}$ be defined by (4.14), and set $C^{+}(\sigma):=\left\{x \in C, \sigma(x)=\mu^{+}\right\}$, for $\sigma \in P_{h}$.

From the result of Appendix A, there exists a function $\mu^{*} \in P_{h}$ such that $\lambda_{1}\left(\mu^{*}\right)=\lambda_{1}$. Let $\Phi^{*} \in H^{1}(C) \subset L^{2 p}(C)$ for some $p>1$, be the eigenfunction associated to the eigenvalue $\lambda_{1}$, with Neumann boundary conditions. Let $P_{h}^{n_{r}, n_{c}}$ be the set of all elements $\sigma_{n_{r}, n_{c}}$ of $P_{h}$ such that $C^{+}\left(\sigma_{n_{r}, n_{c}}\right)$ belongs to the grid of size $n=n_{r} \times n_{c}$. For all $\varepsilon>0$, and for $n_{r}$ and $n_{c}$ large enough, it is easily seen that there exists $\mu_{n_{r}, n_{c}} \in P_{h}^{n_{r}, n_{c}}$ such that $\left\|\mu^{*}-\mu_{n_{r}, n_{c}}\right\|_{L^{p^{\prime}}(C)}<\varepsilon$ (with $\left.\frac{1}{p}+\frac{1}{p^{\prime}}=1\right)$. Let $\Phi_{n_{r}, n_{c}}$ be the principal eigenfunction associated to $\mu_{n_{r}, n_{c}}$ and $\lambda_{1}\left(\mu_{n_{r}, n_{c}}\right)$. Using formula (2.6), and since, when $\mu=\mu^{*}$, the minimum in this formula is precisely attained for $\Phi=\Phi^{*}$, we obtain

$\lambda_{1}\left(\mu_{n_{r}, n_{c}}\right) \leq \lambda_{1}\left(\mu^{*}\right)+\frac{\int_{C}\left|\mu_{n_{r}, n_{c}}-\mu^{*}\right|\left(\Phi^{*}\right)^{2}}{\int_{C}\left(\Phi^{*}\right)^{2}} \leq \lambda_{1}\left(\mu^{*}\right)+\varepsilon\left\|\Phi^{*}\right\|_{L^{2 p}(C)}^{2}\left\|\Phi^{*}\right\|_{L^{2}(C)}^{-2}$,

from Hölder inequality. Setting $\alpha:=\left\|\Phi^{*}\right\|_{L^{2 p}(C)}^{2}\left\|\Phi^{*}\right\|_{L^{2}(C)}^{-2}$ we get $\lambda_{1}\left(\mu_{n_{r}, n_{c}}\right) \leq$ $\lambda_{1}\left(\mu^{*}\right)+\alpha \varepsilon$. Since $P_{h}^{n_{r}, n_{c}} \subset P_{h}$, we therefore have $\lambda_{1}\left(\mu_{n_{r}, n_{c}}\right) \in\left[\lambda_{1}\left(\mu^{*}\right), \lambda_{1}\left(\mu^{*}\right)+\right.$ $\alpha \varepsilon$ ]. Let $\mu_{n_{r}, n_{c}}^{*}$ be a function which minimises $\lambda_{1}$ in $P_{h}^{n_{r}, n_{c}}$; up to a rearrangement we can assume that $\mu_{n_{r}, n_{c}}^{*}$ is nonincreasing with respect to each variable $x_{1}, \cdots, x_{N}$. Then $\lambda_{1}\left(\mu^{*}\right) \leq \lambda_{1}\left(\mu_{n_{r}, n_{c}}^{*}\right) \leq \lambda_{1}\left(\mu_{n_{r}, n_{c}}\right)$; thus, $\lambda_{1}\left(\mu_{n_{r}, n_{c}}^{*}\right) \in$ $\left[\lambda_{1}\left(\mu^{*}\right), \lambda_{1}\left(\mu^{*}\right)+\alpha \varepsilon\right]$, and $\lambda_{1}\left(\mu_{n_{r}, n_{c}}^{*}\right) \rightarrow \lambda_{1}\left(\mu^{*}\right)=\underline{\lambda_{1}}$ as $n \rightarrow \infty$.

From this convergence result, and using the same arguments as in Appendix A, we know that, up to the extraction of some subsequence, $\mu_{n_{r}, n_{c}}^{*} \rightarrow \mu_{\infty}^{*}$ in $L^{1}(C)$ as $n_{r}, n_{c} \rightarrow+\infty$, where $\mu_{\infty}^{*} \in P_{h}$ satisfies $\lambda_{1}\left(\mu_{\infty}^{*}\right)=\underline{\lambda_{1}}$. Let us define the set of "optimal functions" $\mu$ by:

$$
\underline{P}_{h}:=\left\{\mu \in P_{h}, \text { such that } \lambda_{1}(\mu)=\underline{\lambda_{1}}\right\} .
$$

Since we can construct a converging subsequence from every subsequence of $\left(\mu_{n_{r}, n_{c}}^{*}\right)_{n_{r}, n_{c} \in \mathbb{N}}$, the computed configuration $\mu_{n_{r}, n_{c}}^{*}$ is as close as we want to the set $\underline{P}_{h}$ for $n_{r}, n_{c}$ large enough, in the sense that

$$
\operatorname{dist}_{L^{1}(C)}\left(\mu_{n_{r}, n_{c}}^{*}, \underline{P}_{h}\right) \rightarrow 0 \text { as } n_{r}, n_{c} \rightarrow+\infty .
$$

Let $C_{n_{r}, n_{c}}^{+, *}$ be the habitat configuration associated to $\mu_{n_{r}, n_{c}}^{*}$ by (2.4). Then

$$
\operatorname{dist}_{L^{1}(C)}\left(\mu_{n_{r}, n_{c}}^{*}, \underline{P}_{h}\right)=\left|\mu^{+}-\mu^{-}\right| \mathrm{d}_{s e t}\left(C_{n_{r}, n_{c}}^{+, *}, \underline{S}_{h, \mu^{+}, \mu^{-}}\right),
$$

where $\underline{S}_{h, \mu^{+}, \mu^{-}}$is defined by (2.9) and $\mathrm{d}_{s e t}\left(C_{n_{r}, n_{c}}^{+, *}, \underline{S}_{h, \mu^{+}, \mu^{-}}\right)$is defined by (2.102.11). Since $\mu^{+} \neq \mu^{-}, \mathrm{d}_{\text {set }}\left(C_{n_{r}, n_{c}}^{+, *}, \underline{S}_{h, \mu^{+}, \mu^{-}}\right) \rightarrow 0$ as $n_{r}, n_{c} \rightarrow+\infty$. 


\section{Deducing periodic optimal configurations from bounded optimal configurations}

Let $C=\left[0, L_{1}\right] \times \cdots \times\left[0, L_{N}\right], \mu^{+}, \mu^{-} \in \mathbb{R}$, and $h \in[0,1]$. Set $\tilde{C}:=\left[0,2 L_{1}\right] \times \cdots \times$ $\left[0,2 L_{N}\right]$. Let $P_{h}$ be defined by $(4.14)$, and let $\tilde{P}_{h}$ be the set of functions defined by (4.14) but with $\tilde{C}$ instead of $C$. For a function $\sigma$ in $\tilde{P}_{h}$, we denote by $\tilde{C}^{+}(\sigma):=$ $\left\{x \in \tilde{C}, \sigma(x)=\mu^{+}\right\}$. Let $\lambda_{1, b}(\mu)$ denote the Neumann principal eigenvalue of $-D \nabla^{2}-\mu$ in $C$, and $\lambda_{1, p}(\sigma)$ denote the periodic principal eigenvalue of $-D \nabla^{2}-\sigma$ in $\tilde{C}$.

From Appendix A, we know that there exists a function $\mu_{b}^{*}$ in $\underline{P}_{h}$, nonincreasing with respect to each variable $x_{1}, \cdots, x_{N}$. Let us show that $\mu_{p}^{*}\left(x_{1}, \cdots, x_{N}\right):=$ $\mu_{b}^{*}\left(\left|x_{1}-L_{1}\right|, \cdots,\left|x_{N}-L_{N}\right|\right)$ minimises $\lambda_{1, p}$ in $\tilde{P}_{h}$.

Indeed, let us set $\underline{\lambda_{1, b}}:=\min _{\sigma \in P_{h}} \lambda_{1, b}(\sigma)$ and $\underline{\lambda_{1, p}}:=\min _{\sigma \in \tilde{P}_{h}} \lambda_{1, p}(\sigma)$, and

$$
\underline{\tilde{P}}_{h}:=\left\{\mu \in \tilde{P}_{h}, \text { such that } \lambda_{1, p}(\mu)=\underline{\lambda_{1, p}}\right\} .
$$

Let $\mu_{p}$ be a symmetric nonincreasing function in $\underline{\tilde{P}}_{h}$ and $\underline{\Psi}_{p}$ be the corresponding symmetric nonincreasing eigenfunction (see [25]). Set

$$
\underline{\Psi_{b}}\left(x_{1}, \cdots, x_{N}\right):=\underline{\Psi_{p}}\left(x_{1}+L_{1}, \cdots, x_{N}+L_{N}\right) .
$$

The function $\Psi_{b}$ is well defined on $C$, and by symmetry and periodicity properties of $\Psi_{p}$, it is of class $C^{1}(\bar{C})$ and it satisfies Neumann boundary conditions. Moreover, $\underline{\Psi_{b}}>0$,

$$
-D \nabla^{2} \underline{\Psi_{b}}-\underline{\mu_{p}}\left(x_{1}+L_{1}, \cdots, x_{N}+L_{N}\right) \underline{\Psi_{b}}=\underline{\lambda_{1, p}} \underline{\Psi_{b}} \text { in } C,
$$

and $\mu_{p}\left(\cdot+L_{1}, \cdots, \cdot+L_{N}\right) \in P_{h}$. By definition of $\lambda_{1, b}$, we get $\lambda_{1, p} \geq \lambda_{1, b}$.

Let $\Phi_{b}$ be the eigenfunction corresponding to $\overline{\lambda_{1, b}}$ with the configuration $\mu_{b}^{*}$. Let us define

$$
\underline{\Phi_{p}}\left(x_{1}, \cdots, x_{N}\right):=\underline{\Phi_{b}}\left(\left|x_{1}-L_{1}\right|, \cdots,\left|x_{N}-L_{N}\right|\right) \text { in } \tilde{C}
$$

and let $\mu_{p}^{*}\left(x_{1}, \cdots, x_{N}\right)=\mu_{b}^{*}\left(\left|x_{1}-L_{1}\right|, \cdots,\left|x_{N}-L_{N}\right|\right)$. Then, from the Neumann boundary conditions satisfied by $\Phi_{b}$, it follows that $\Phi_{p}$ is a weak solution of $-D \nabla^{2} \underline{\Phi_{p}}-\mu_{p}^{*}(x) \underline{\Phi_{p}}=\underline{\lambda_{1, b} \Phi_{p}}$ in $\overline{\tilde{C}}$ with periodic boundary conditions. Since

$$
\left|\tilde{C}^{+}\left(\mu_{p}^{*}\right)\right|=\frac{1}{\mu^{+}} \int_{\tilde{C} \cap\left\{\mu_{p}^{*}=\mu^{+}\right\}} \mu_{p}^{*}=\frac{2^{N}}{\mu^{+}} \int_{C \cap\left\{\mu_{b}^{*}=\mu^{+}\right\}} \mu_{b}^{*}=2^{N}\left|C^{+}\left(\mu_{b}^{*}\right)\right|=h|\tilde{C}|,
$$

it follows that $\mu_{p}^{*} \in \tilde{P}_{h}$. Thus, we get $\lambda_{1, p} \leq \lambda_{1, b}$, and finally $\lambda_{1, p}=\lambda_{1, b}$. As a conclusion, we obtain that $\mu_{p}^{*} \in \underline{\tilde{P}}_{h}$, and therefore that $\tilde{C}^{+}\left(\mu_{p}^{*}\right)$ is an optimal habitat configuration in the periodic case, with period cell $\tilde{C}$. Note that $\tilde{C}^{+}\left(\mu_{p}^{*}\right)$ simply corresponds to successive reflections with respect to each axis $\left\{x_{i}=L_{i}\right\}$, for $i=1 \ldots N$ starting from the optimal configuration $C^{+}\left(\mu_{b}^{*}\right)$ in the bounded case. 


\section{Proof of the comparison principle between the striped configurations}

The proof is done in the periodic case. Let $\lambda_{V}$ be the value of $\lambda_{1}$ in the case of the vertical configuration depicted in Fig. 3 (a), and $\lambda_{H}$ be the value of $\lambda_{1}$ for the horizontal configuration depicted in Fig. 3 (b). Then, a simple change of variable leads to $\lambda_{V}\left[L_{1}, L_{2}\right]=\lambda_{H}\left[L_{2}, L_{1}\right]$ (in $\lambda_{V}[X, Y]$ and $\lambda_{H}[X, Y], X$ corresponds to the length of the period cell in the $x_{1}$ direction and $Y$ corresponds to the length of the period cell in the $x_{2}$ direction). Since $L_{1} L_{2}=|C|$, we get

$$
\lambda_{V}\left[L_{1},|C| / L_{1}\right]=\lambda_{H}\left[|C| / L_{1}, L_{1}\right] .
$$

Let us now prove that $\lambda_{V}\left[L_{1},|C| / L_{1}\right]$ decreases with $L_{1}$. Assume that we are in the configuration of the vertical stripe. Then $\mu\left(x_{1}, x_{2}\right)$ does not depend on $x_{2}$. Set $\tilde{\mu}\left(x_{1}\right):=\mu\left(x_{1}, 0\right)$. The function $\tilde{\mu}$ is $L_{1}$-periodic and, in the period cell $\left[0, L_{1}\right], \tilde{\mu}$ is equal to $\mu^{+}$on an interval of length $l_{1}=\left|C^{+}\right| / L_{2}$, and it is equal to $\mu^{-}$on an interval of length $L_{1}-l_{1}$. Let $\Phi$ and $\lambda_{V}=\lambda_{1}$ be defined by equation (2.5). Actually, since $\Phi$ is uniquely determined by (2.5) and the periodicity conditions, it depends on $x_{1}$ only. Set $\psi\left(x_{1}\right):=\Phi\left(x_{1}, 0\right)$. Then $\psi$ and $\lambda_{V}$ satisfy the following one-dimensional equation:

$$
-D \psi^{\prime \prime}\left(x_{1}\right)-\tilde{\mu}\left(x_{1}\right) \psi\left(x_{1}\right)=\lambda_{V} \psi\left(x_{1}\right), x_{1} \in \mathbb{R}, \psi>0 \text { in } \mathbb{R}
$$

and $\psi$ is $L_{1}$-periodic. Now, set $\hat{\mu}(y):=\tilde{\mu}\left(y L_{1}\right)$. The function $\hat{\mu}$ is 1 -periodic and, in the period cell $[0,1], \hat{\mu}$ is equal to $\mu^{+}$on an interval of length $l_{1}^{\prime}=$ $l_{1} / L_{1}=\left|C^{+}\right| /\left(L_{1} L_{2}\right)=\left|C^{+}\right| /|C| \in(0,1)$, and it is equal to $\mu^{-}$on an interval of length $1-l_{1}^{\prime}$. The function $\hat{\mu}$ does not depend on $L_{1}$. The 1-periodic function $\xi$ defined by $\xi(y)=\psi\left(y L_{1}\right)$ satisfies

$$
-\left(L_{1}\right)^{-2} D \xi^{\prime \prime}(y)-\hat{\mu}(y) \xi(y)=\lambda_{V} \xi(y), y \in \mathbb{R}, \xi>0 \text { in } \mathbb{R} .
$$

Then, since the operator $\varphi \longmapsto-\left(L_{1}\right)^{-2} D \varphi^{\prime \prime}-\hat{\mu} \varphi$ is self-adjoint, we have the following formula:

$$
\lambda_{V}=\min _{\varphi \in H_{p e r}^{1} \backslash\{0\}}\left[\frac{1}{L_{1}^{2}} \times \frac{\int_{0}^{1} D\left(\varphi^{\prime}\right)^{2}(y) d y}{\int_{0}^{1} \varphi^{2}(y) d y}-\frac{\int_{0}^{1} \hat{\mu}(y) \varphi^{2}(y) d y}{\int_{0}^{1} \varphi^{2}(y) d y}\right],
$$

where $H_{\text {per }}^{1}$ denotes here the set of $H_{\text {loc }}^{1}(\mathbb{R})$ functions which are 1-periodic. From (4.25), we get that $\lambda_{V}=\lambda_{V}\left[L_{1},|C| / L_{1}\right]$ is decreasing with respect to $L_{1}$. Notice that the strict monotonicity follows from the fact that the minimum in (4.25) is reached at the function $\xi$, which is not constant since $\hat{\mu}$ is not constant. As a conclusion, using (4.22), it follows that $\lambda_{V}\left[L_{1}, L_{2}\right]>\lambda_{H}\left[L_{1}, L_{2}\right]$ if $L_{1}<L_{2}$. 


\section{E. Dependence of the optimal configuration on the amplitude of the variations of the environ- ment}

We consider here the periodic case. We fix $N \geq 2$. In the sequel, for any Borel set $C^{+} \subset C^{\circ}:=\left(0, L_{1}\right) \times \cdots \times\left(0, L_{N}\right)$ and for any real numbers $\mu^{-}<\mu^{+}$, we call

$$
\lambda_{1}\left[C^{+}, \mu^{+}, \mu^{-}\right]=\min _{\varphi \in H_{\text {per }}^{1} \backslash\{0\}} \frac{\int_{C} D|\nabla \varphi|^{2}-\int_{C} \mu(x) \varphi^{2}}{\int_{C} \varphi^{2}}
$$

the principal eigenvalue of the operator $-D \nabla^{2}-\mu(x)$ in $\mathbb{R}^{N}$ with $L$-periodicity conditions, where $\mu(x)=\mu^{+}$in $C^{+}, \mu(x)=\mu^{-}$in $C^{-}=C \backslash C^{+}$, and $\mu$ is extended in $\mathbb{R}^{N}$ by $L$-periodicity.

Integrating equation (2.5) over the cell $C$ and using the periodicity of $\Phi$, we get

$$
-\int_{C} \mu(x) \Phi(x) d x=\lambda_{1}\left[C^{+}, \mu^{+}, \mu^{-}\right] \int_{C} \Phi(x) d x .
$$

Since $\mu \geq \mu^{-}$, it follows that $\lambda_{1}\left[C^{+}, \mu^{+}, \mu^{-}\right] \leq-\mu^{-}$. Next, multiply (2.5) by $\Phi$ and integrate by parts over $C$, we get

$$
\int_{C} D|\nabla \Phi|^{2}(x) d x-\int_{C} \mu(x) \Phi^{2}(x) d x=\lambda_{1}\left[C^{+}, \mu^{+}, \mu^{-}\right] \int_{C} \Phi^{2}(x) d x,
$$

and since $\mu \leq \mu^{+}$, we get $\lambda_{1}\left[C^{+}, \mu^{+}, \mu^{-}\right] \geq-\mu^{+}$. Finally,

$$
-\mu^{+} \leq \lambda_{1}\left[C^{+}, \mu^{+}, \mu^{-}\right] \leq-\mu^{-} .
$$

Step 1: limit of $\lambda_{1}\left[C^{+}, 0,-n\right]$ when $C^{+}$is fixed and $n \rightarrow+\infty$. Let $C^{+}$be a fixed Borel subset of $C^{\circ}$ whose $N$-dimensional Lebesgue measure belongs to the interval $(0,|C|)$. For $n \in \mathbb{N} \backslash\{0\}$, call $\mu_{n}$ the $L$-periodic function such that

$$
\mu_{n}(x)=0 \text { if } x \in C^{+} \text {, and } \mu_{n}(x)=-n \text { if } x \in C^{-}=C \backslash C^{+} .
$$

Call $\lambda_{1, \mathcal{D}}\left[C^{+}\right]$the quantity defined by

$$
\lambda_{1, \mathcal{D}}\left[C^{+}\right]=\min _{\varphi \in H_{0, p e r}^{1}\left(C^{+}\right) \backslash\{0\}} \frac{\int_{C^{+}} D|\nabla \varphi|^{2}}{\int_{C^{+}} \varphi^{2}},
$$

where $H_{0, p e r}^{1}\left(C^{+}\right)$denotes the set of $H_{p e r}^{1}$ functions which vanish almost everywhere in $C^{-}$. When $C^{+}$is a smooth open set, $\lambda_{1, \mathcal{D}}$ is the principal eigenvalue of the operator $-D \nabla^{2}$ in $C^{+}$with Dirichlet boundary condition on $\partial C^{+} \cap C$ and $L$-periodicity. Since $\mu_{n}=0$ in $C^{+}$, it follows from (4.28) and (4.26) with $\mu=\mu_{n}$ that

$$
\lambda_{1}\left[C^{+}, 0,-n\right] \leq \lambda_{1, \mathcal{D}}\left[C^{+}\right]
$$


for all $n \in \mathbb{N} \backslash\{0\}$. Call $\Phi_{n}$ the principal eigenfunction of the operator $-D \nabla^{2}-$ $\mu_{n}$ with $L$-periodicity, with the normalisation $\left\|\Phi_{n}\right\|_{L^{2}(C)}=1$. One has

$$
\lambda_{1}\left[C^{+}, 0,-n\right]=\int_{C} D\left|\nabla \Phi_{n}\right|^{2}+n \int_{C^{-}} \Phi_{n}^{2} .
$$

From (4.29) and (4.30), it follows that the sequence $\left(\left\|\nabla \Phi_{n}\right\|_{L^{2}(C)}\right)_{n \in \mathbb{N} \backslash\{0\}}$ is bounded and that $\left\|\Phi_{n}\right\|_{L^{2}\left(C^{-}\right)} \rightarrow 0$ as $n \rightarrow+\infty$. Therefore, there exist a subsequence $\left(\Phi_{n^{\prime}}\right)$ and a function $\varphi \in H_{0, p e r}^{1}\left(C^{+}\right)$such that $\Phi_{n^{\prime}} \rightarrow \Phi$ as $n^{\prime} \rightarrow$ $+\infty$ strongly in $L_{l o c}^{2}\left(\mathbb{R}^{N}\right)$ and weakly in $H_{l o c}^{1}\left(\mathbb{R}^{N}\right)$. In particular, $\|\Phi\|_{L^{2}\left(C^{+}\right)}=$ $\|\Phi\|_{L^{2}(C)}=1$. Furthermore, since $\lambda_{1}\left[C^{+}, 0,-n^{\prime}\right] \geq D\left\|\nabla \Phi_{n^{\prime}}\right\|_{L^{2}(C)}^{2}$, it follows that

$$
\liminf _{n^{\prime} \rightarrow+\infty} \lambda_{1}\left[C^{+}, 0,-n^{\prime}\right] \geq D\|\nabla \Phi\|_{L^{2}(C)}^{2}=D\|\nabla \Phi\|_{L^{2}\left(C^{+}\right)}^{2} .
$$

As a consequence, $\liminf _{n^{\prime} \rightarrow+\infty} \lambda_{1}\left[C^{+}, 0,-n^{\prime}\right] \geq \lambda_{1, \mathcal{D}}\left[C^{+}\right]$from (4.28), whence $\lambda_{1}\left[C^{+}, 0,-n^{\prime}\right] \rightarrow \lambda_{1, \mathcal{D}}\left[C^{+}\right]$as $n^{\prime} \rightarrow+\infty$, from (4.29). Eventually, since the limit is uniquely determined, one concludes that the whole sequence converges, that is

$$
\lambda_{1}\left[C^{+}, 0,-n\right] \rightarrow \lambda_{1, \mathcal{D}}\left[C^{+}\right] \text {as } n \rightarrow+\infty .
$$

Step 2: A pointwise inequality for the principal eigenfunction associated to an optimal configuration. Let $\mu^{-}<\mu^{+}$and $h \in(0,1)$ be fixed. From Appendix A, there exists a Borel set $C^{+} \subset C^{\circ}$ such that $C^{+} \in \underline{S}_{h, \mu^{+}, \mu^{-}}\left(\underline{S}_{h, \mu^{+}, \mu^{-}}\right.$is defined by (2.9)). Notice that the set $C^{+}$could be chosen to be symmetric with respect to the hyperplanes $\left\{x_{i}=L_{i} / 2\right\}$ for all $1 \leq i \leq N$, but this property is not needed here. Call $\mu$ the $L$-periodic function which is equal to $\mu^{+}$in $C^{+}$and to $\mu^{-}$in $C^{-}=C \backslash C^{+}$. Let $\Phi$ be the (unique up to multiplication) principal eigenfunction of the operator $-D \nabla^{2}-\mu$ with $L$-periodicity. The function $\Phi$ is positive, it belongs to all Sobolev spaces $W_{l o c}^{2, p}\left(\mathbb{R}^{N}\right)$ for $1 \leq p<+\infty$ (in particular, it is of class $C^{1, \alpha}\left(\mathbb{R}^{N}\right)$ for all $\left.0 \leq \alpha<1\right)$ and it satisfies

$$
-D \nabla^{2} \Phi-\mu(x) \Phi=\lambda_{1}\left[C^{+}, \mu^{+}, \mu^{-}\right] \Phi .
$$

The goal of this step is to prove that

$$
\inf _{\left(C^{+}\right)^{\circ}} \Phi=\sup _{\left(C^{-}\right)^{\circ}} \Phi
$$

It is enough to prove that

$$
\Phi\left(x_{0}\right) \geq \Phi\left(y_{0}\right)
$$

for all $x_{0}$ and $y_{0}$ which are interior points of $C^{+}$and $C^{-}$respectively. There exists $\varepsilon_{0}>0$ such that $B_{\varepsilon}\left(x_{0}\right)$ (resp. $\left.B_{\varepsilon}\left(y_{0}\right)\right)$ is included in the interior of $C^{+}$ (resp. in the interior of $C^{-}$) for all $0<\varepsilon<\varepsilon_{0}$, where $B_{r}(x)$ denotes the open Euclidean ball of centre $x$ and radius $r>0$. For $\varepsilon \in\left(0, \varepsilon_{0}\right)$, call

$$
C_{\varepsilon}^{+}=\left(C^{+} \backslash B_{\varepsilon}\left(x_{0}\right)\right) \cup B_{\varepsilon}\left(y_{0}\right) \text { and } C_{\varepsilon}^{-}=C \backslash C_{\varepsilon}^{+} .
$$


By construction, the Borel set $C_{\varepsilon}^{+}$is included in $C^{\circ}$ and its Lebesgue measure is equal to $h|C|$. Let $\mu_{\varepsilon}$ be the $L$-periodic function which is equal to $\mu^{+}$in $C_{\varepsilon}^{+}$and to $\mu^{-}$in $C_{\varepsilon}^{-}$, and let $\Phi_{\varepsilon}$ be the principal eigenfunction of $-D \nabla^{2}-\mu_{\varepsilon}$ with $L$ periodicity. Call $\lambda=\lambda_{1}\left[C^{+}, \mu^{+}, \mu^{-}\right]$and $\lambda_{\varepsilon}=\lambda_{1}\left[C_{\varepsilon}^{+}, \mu^{+}, \mu^{-}\right]\left(\right.$for $\left.0<\varepsilon<\varepsilon_{0}\right)$. By definition of $\lambda$, there holds

$$
\lambda \leq \lambda_{\varepsilon}
$$

The functions $\Phi$ and $\Phi_{\varepsilon}$ are positive and $L$-periodic solutions of

$$
\left\{\begin{array}{rll}
-D \nabla^{2} \Phi-\mu(x) \Phi & = & \lambda \Phi \\
-D \nabla^{2} \Phi_{\varepsilon}-\mu_{\varepsilon}(x) \Phi_{\varepsilon} & = & \lambda_{\varepsilon} \Phi_{\varepsilon}
\end{array} \quad \text { in } \mathbb{R}^{N}\right.
$$

and up to normalisation, one can assume that $\|\Phi\|_{\infty}=\left\|\Phi_{\varepsilon}\right\|_{\infty}=1$. The families $\left(\lambda_{\varepsilon}\right)_{0<\varepsilon<\varepsilon_{0}}$ and $\left(\mu_{\varepsilon}\right)_{0<\varepsilon<\varepsilon_{0}}$ are bounded in $\mathbb{R}$ and $L^{\infty}\left(\mathbb{R}^{N}\right)$ respectively. From standard elliptic estimates, the family $\left(\Phi_{\varepsilon}\right)_{0<\varepsilon<\varepsilon_{0}}$ is then bounded in all $W_{l o c}^{2, p}\left(\mathbb{R}^{N}\right)$ spaces with $1 \leq p<+\infty$. Standard arguments (see for instance [25]) imply then that $\lambda_{\varepsilon} \rightarrow \lambda$ and $\Phi_{\varepsilon} \rightarrow \Phi$ in $C^{1}\left(\mathbb{R}^{N}\right)$ (at least) as $\varepsilon \rightarrow 0$. Multiply now the first equation of (4.34) by $\Phi_{\varepsilon}$ and the second one by $\Phi$ and subtract and integrate over $C$ the two quantities. It follows that

$$
-\int_{C}\left(\mu-\mu_{\varepsilon}\right) \Phi \Phi_{\varepsilon}=\left(\lambda-\lambda_{\varepsilon}\right) \int_{C} \Phi \Phi_{\varepsilon} \leq 0
$$

from (4.33) and the positivity of $\Phi$ and $\Phi_{\varepsilon}$. In $C$, the function $\mu-\mu_{\varepsilon}$ is equal to $\mu^{+}-\mu^{-}$in $B_{\varepsilon}\left(x_{0}\right)$, to $\mu^{-}-\mu^{+}$in $B_{\varepsilon}\left(y_{0}\right)$ and to 0 otherwise. Since $\mu^{+}>\mu^{-}$, it follows that

$$
\int_{B_{\varepsilon}\left(x_{0}\right)} \Phi \Phi_{\varepsilon} \geq \int_{B_{\varepsilon}\left(y_{0}\right)} \Phi \Phi_{\varepsilon} .
$$

By dividing the previous inequality by $\alpha_{N} \varepsilon^{N}$ (where $\alpha_{N}$ denotes the Lebesgue measure of the unit Euclidean ball in $\mathbb{R}^{N}$ ) and passing to the limit as $\varepsilon \rightarrow 0$, one concludes that $\Phi\left(x_{0}\right)^{2} \geq \Phi\left(y_{0}\right)^{2}$. Since $\Phi$ is positive, the inequality (4.32) follows and the claim (4.31) is proved.

Notice that the inequality (4.31) implies in particular that the function $\Phi$ is constant on $\partial\left(C^{+}\right)^{\circ} \cap \partial\left(C^{-}\right)^{\circ}$. This property was observed in the numerical computations which were carried out in Section 3.

Step 3: Conclusion. Up to rotation of the frame, one can assume that $L_{1}=$ $\min \left(L_{1}, \ldots, L_{N}\right)$. Call $r=L_{1} / 2$ and $c=\left(L_{1} / 2, \ldots, L_{N} / 2\right)$ the centre of the cell $C$. The ball $B_{r}(c)$ is included in $C$ and it is tangent to the two hyperplanes $\left\{x_{1}=0\right\}$ and $\left\{x_{1}=L_{1}\right\}$, like the black region in Fig. 6. Call $\alpha_{N} r^{N}$ the Lebesgue measure of this ball, and assume that

$$
h=\frac{\alpha_{N} r^{N}}{|C|} .
$$

Assume now that there is a Borel set $C^{+} \subset C^{\circ}$ such that $C^{+} \in \underline{S}_{h, 0,-n}$ for all $n \in \mathbb{N} \backslash\{0\}$. In other words, we assume that

$$
\lambda_{1}\left[C^{+}, 0,-n\right]=\min _{K \in S_{h}} \lambda_{1}[K, 0,-n] \text { for all } n \in \mathbb{N} \backslash\{0\} .
$$


From this assumption, we claim that there exist a point $x \in C$ and a zeromeasure set $E$ such that

$$
C^{+} \subset B_{r}(x) \cup E .
$$

If not, since the ball $B_{r}(c)$ has the same measure as $C^{+}$and is included in $C$, it follows from the definition of $\lambda_{1, \mathcal{D}}\left[C^{+}\right]$in Step 1 and from Schwarz rearrangement inequalities that

$$
\lambda_{1, \mathcal{D}}\left[C^{+}\right]>\lambda_{1, \mathcal{D}}\left[B_{r}(c)\right] .
$$

Indeed, let $\Phi$ be a minimiser of (4.28), call $\Phi$ its restriction in $C$ and call $B_{R}(c)$ the open ball of centre $c$ and radius $R>r$ which has the same measure as $C$. The Schwarz decreasing symmetric rearrangement $\Phi^{*}$ of $\Phi$ with respect to the point $c$ is in $H_{0}^{1}\left(B_{R}(c)\right)$ and it is such that $\Phi^{*}=0$ in $B_{R}(c) \backslash B_{r}(c)$ and

$$
\frac{\int_{B_{r}(c)} D\left|\nabla \Phi^{*}\right|^{2}}{\int_{B_{r}(c)}\left(\Phi^{*}\right)^{2}}<\frac{\int_{C^{+}} D|\nabla \Phi|^{2}}{\int_{C^{+}} \Phi^{2}}=\lambda_{1, \mathcal{D}}\left[C^{+}\right]
$$

since $C^{+}$is not included in any ball of radius $r$, up to a zero-measure set. Notice in particular that the restriction of $\Phi^{*}$ to $B_{r}(c)$ is in $H_{0}^{1}\left(B_{r}(c)\right)$. Now define $\bar{\Phi}^{*}=\Phi^{*}$ in $B_{r}(c)$ and $\bar{\Phi}^{*}=0$ in $C \backslash B_{r}(c)$, and extend $\bar{\Phi}^{*}$ in $\mathbb{R}^{N}$ by $L$-periodicity. The function $\bar{\Phi}^{*}$ is then in $H_{0, p e r}^{1}\left(B_{r}(c)\right) \backslash\{0\}$, whence

$$
\lambda_{1, \mathcal{D}}\left[B_{r}(c)\right] \leq \frac{\int_{B_{r}(c)} D\left|\nabla \bar{\Phi}^{*}\right|^{2}}{\int_{B_{r}(c)}\left(\bar{\Phi}^{*}\right)^{2}}=\frac{\int_{B_{r}(c)} D\left|\nabla \Phi^{*}\right|^{2}}{\int_{B_{r}(c)}\left(\Phi^{*}\right)^{2}}<\lambda_{1, \mathcal{D}}\left[C^{+}\right],
$$

which yields (4.36). Step 1 yields $\lambda_{1}\left[C^{+}, 0,-n\right]>\lambda_{1}\left[B_{r}(c), 0,-n\right]$ for $n$ large enough, which is in contradiction with (4.35), since $B_{r}(c)$ is included in $C$ and has the same measure $h|C|$ as $C^{+}$.

As a consequence, the set $C^{+}$is included in a ball of radius $r$, up to a set of zero measure. Up to a shift and a modification with a zero-measure set, one can then assume without loss of generality that

$$
C^{+}=B_{r}(c) .
$$

Fix now any $n_{0} \in \mathbb{N} \backslash\{0\}$. Call $\mu$ the $L$-periodic function which is equal to 0 in $B_{r}(c)$ and to $-n_{0}$ in $C \backslash B_{r}(c)$, and call $\Phi$ the principal eigenfunction of the operator $-D \nabla^{2}-\mu$ with $L$-periodicity. Call $\lambda=\lambda_{1}\left[B_{r}(c), 0,-n_{0}\right]$ the principal eigenvalue. The function $\Phi$ is (at least) of class $C^{1}\left(\mathbb{R}^{N}\right)$ and it is positive in $\mathbb{R}^{N}$. From Step 2, the function $\Phi$ is equal to a constant $\beta>0$ on $\partial B_{r}(c)$, and it is larger than or equal to $\beta$ in $B_{r}(c)$. In $B_{r}(c)$, the function $\Phi$ solves $-D \nabla^{2} \Phi=\lambda \Phi$ and the function $\psi=\Phi-\beta$ satisfies

$$
\left\{\begin{aligned}
-D \nabla^{2} \psi-\lambda \psi & =\lambda \beta>0 & & \text { in } B_{r}(c), \\
\psi & \geq 0 & & \text { in } B_{r}(c), \\
\psi & =0 & & \text { on } \partial B_{r}(c)
\end{aligned}\right.
$$


since $0<\lambda\left(<n_{0}\right)$. It follows from the strong maximum principle and Hopf lemma that $\psi>0$ in $B_{r}(c)$ and

$$
\frac{\partial \psi}{\partial x_{1}}(a)>0
$$

where $a=\left(0, L_{2} / 2, \ldots, L_{N} / 2\right) \in \partial B_{c}(r)$. Therefore, the partial derivative of $\Phi$ with respect to $x_{1}$ at the point $a$ is positive. But since the function $\mu$ is symmetric with respect to $x_{1}$ in $\mathbb{R}^{N}$ (because $r=L_{1} / 2$, see Fig. 6 ), the function $\Phi$ is also symmetric with respect to $x_{1}$, by uniqueness. Therefore, $\frac{\partial \Phi}{\partial x_{1}}(a)=0$, a contradiction has been reached.

As a conclusion, the assumption (4.35) cannot hold, which means that the optimal habitat configurations depend on the difference between the growth rates in habitat and non-habitat regions in general.

Notice that, under the notations in Step 3, the above arguments imply that the ball $B_{r}(c)$ is never in $\underline{S}_{h, \mu^{+}, \mu^{-}}$, for all $\mu^{+}>\mu^{-}$, when $h=\frac{\alpha_{N} r^{N}}{|C|}$. In particular, this shows rigourously that ball-shaped configurations are not always optimal for species persistence.

Remark 4.1 In the bounded case, under the assumptions of Proposition 3.2, if we assume that $\lambda_{1}\left[C^{+}\right]=\lambda_{1}$, it follows from Step 2 that $\Phi(x)>\Phi\left(x_{0}\right)$ for all $x \in B^{\circ}$. Then, as in Step 3 , applying Hopf's lemma at the point $x_{0}$, we get that $\frac{\partial \Phi}{\partial n}\left(x_{0}\right)<0$. This is in contradiction with the boundary conditions.

\section{F. Stripe-shaped configurations are not always optimal}

We prove here that stripe-shaped configurations are not always optimal for species persistence. The proof is done in the periodic case, in dimension $N=2$, but it can be adapted immediately in higher dimensions.

Fix two real numbers $\mu^{-}<\mu^{+}$. Call $\lambda_{\mathcal{D}}\left(B_{R}(x)\right)$ the principal eigenvalue of $-D \nabla^{2}$ in the ball $B_{R}(x)$ with Dirichlet boundary conditions on $\partial B_{R}(x)$. Since $\lambda_{\mathcal{D}}\left(B_{R}(x)\right)=R^{-2} \lambda_{\mathcal{D}}\left(B_{1}(0)\right)$, we can fix $R>0$ large enough such that

$$
\lambda_{\mathcal{D}}\left(B_{R}(x)\right)<\frac{\mu^{+}-\mu^{-}}{2}, \text { for all } x \in \mathbb{R}^{2}
$$

Call $A^{+}=\pi R^{2}$, fix two positive real numbers $l_{1}$ and $l_{2}$, and a positive real number $\beta_{0}$ such that $\beta_{0}^{2} l_{1} l_{2}>A^{+}$. We will compare the two stripe-configurations which are depicted in Fig. 3, where $L_{1}=\beta l_{1}$ and $L_{2}=\beta l_{2}$, with the one for which the habitat is a disc a radius $R$ in the cell $C=C_{\beta}=\left[0, L_{1}\right] \times\left[0, L_{2}\right]$, for $\beta$ large. The two stripe-configurations and the disc-shaped configurations are constructed so that the habitat be of fixed area $A^{+}$in $C$. We will prove that the disc-shaped habitat is better when $\beta$ is large.

Pick any $\beta \geq \beta_{0}$, and let $x$ be a point such that $B_{R}(x) \subset\left[0, L_{1}\right] \times\left[0, L_{2}\right]$. From [47], it is known that $\lambda_{1}\left[B_{R}(x), \mu^{+}, \mu^{-}\right]$is less than the principal eigenvalue 
of the operator $-D \nabla^{2}-\mu$ in the ball $B_{R}(x)$ with Dirichlet boundary condition on $\partial B_{R}(x)$. Since $-\mu=-\mu^{+}$in the habitat $B_{R}(x)$, it follows that

$$
\lambda_{1}\left[B_{R}(x), \mu^{+}, \mu^{-}\right]<\lambda_{\mathcal{D}}\left(B_{R}(x)\right)-\mu^{+}<-\frac{\mu^{+}+\mu^{-}}{2}
$$

from our choice of $R$ in (4.37).

Let us now estimate $\lambda_{\beta}:=\lambda_{V}\left[L_{1}, L_{2}\right]=\lambda_{V}\left[\beta l_{1}, \beta l_{2}\right]$, for $\beta \geq \beta_{0}$. From the arguments used in Appendix E, there exists a positive function $\psi_{\beta}$ defined in $\mathbb{R}$, which is $L_{1}$-periodic and satisfies

$$
-D \psi_{\beta}^{\prime \prime}-\mu_{\beta}\left(x_{1}\right) \psi_{\beta}=\lambda_{\beta} \psi_{\beta} \text { almost everywhere in } \mathbb{R}
$$

where the function $\mu_{\beta}$ is $L_{1}$-periodic and, up to shift, one can assume without loss of generality that

$$
\left\{\begin{array}{l}
\mu_{\beta}\left(x_{1}\right)=\mu^{+} \quad \text { if } 0 \leq x_{1} \leq A^{+} / L_{2}=A^{+} /\left(\beta l_{2}\right) \\
\mu_{\beta}\left(x_{1}\right)=\mu^{-} \quad \text { if } A^{+} /\left(\beta l_{2}\right)<x_{1}<L_{1}=\beta l_{1}
\end{array}\right.
$$

The function $\psi_{\beta}$ is in all spaces $W_{\text {loc }}^{2, p}(\mathbb{R})$ for $1 \leq p<+\infty$ and one can assume, up to normalisation, that $\left\|\psi_{\beta}\right\|_{\infty}=1$. Furthermore, $\psi_{\beta}$ is analytic in the open intervals $\left(0, A^{+} /\left(\beta l_{2}\right)\right)$ and $\left(A^{+} /\left(\beta l_{2}\right), L_{1}\right)$. If $\psi_{\beta}$ reaches its maximum at a point $x_{0} \in\left(A^{+} /\left(\beta l_{2}\right), L_{1}\right)$, then $-\psi_{\beta}^{\prime \prime}\left(x_{0}\right) \geq 0$, whence $\lambda_{\beta} \geq-\mu_{\beta}\left(x_{0}\right)=-\mu^{-}$, which is impossible since $-\mu^{+}<\lambda_{\beta}<-\mu^{-}$. Therefore, the maximum of $\psi_{\beta}$ is reached in the interval $\left[0, A^{+} /\left(\beta l_{2}\right)\right]{ }^{1}$ Since the families $\left(\lambda_{\beta}\right)_{\beta \geq \beta_{0}}$ and $\left(\mu_{\beta}\right)_{\beta \geq \beta_{0}}$ are bounded in $\mathbb{R}$ and $L^{\infty}(\mathbb{R})$ respectively, the functions $\left(\bar{\psi}_{\beta}\right)_{\beta \geq \beta_{0}}$ are bounded in all $W_{l o c}^{2, p}(\mathbb{R})$ for $1 \leq p<+\infty$. There exist then a sequence $\beta_{n} \rightarrow+\infty$ and a function $\psi_{\infty}$ which is in all $W_{l o c}^{2, p}(\mathbb{R})$ for $1 \leq p<+\infty$, such that $\lambda_{\beta_{n}} \rightarrow \lambda_{\infty} \in\left[-\mu^{+},-\mu^{-}\right]$and the functions $\psi_{\beta_{n}}$ converge (at least) in $C_{\text {loc }}^{1}(\mathbb{R})$ to $\psi_{\infty}$ as $n \rightarrow+\infty$. Furthermore, since $\mu_{\beta_{n}}(x) \rightarrow \mu^{-}$almost everywhere in $\mathbb{R}$ (the convergence holds for all $x \neq 0$ ), the function $\psi_{\infty}$ satisfies

$$
-D \psi_{\infty}^{\prime \prime}-\mu^{-} \psi_{\infty}=\lambda_{\infty} \psi_{\infty} \text { almost everywhere in } \mathbb{R} .
$$

The function $\psi_{\infty}$ is then of class $C^{2}(\mathbb{R})$. Furthermore, $\psi_{\infty}(0)=1=\max _{\mathbb{R}} \psi_{\infty}$, whence $\lambda_{\infty} \geq-\mu^{-}$. As a consequence, $\lambda_{\infty}=-\mu^{-}$and by uniqueness of the limit, the whole family $\left(\lambda_{\beta}=\lambda_{V}\left[\beta l_{1}, \beta l_{2}\right]\right)_{\beta \geq \beta_{0}}$ converges to $-\mu^{-}$as $\beta \rightarrow+\infty$. Similarly, $\lambda_{H}\left[\beta l_{1}, \beta l_{2}\right] \rightarrow-\mu^{-}$as $\beta \rightarrow+\infty$.

Together with (4.38), we conclude that

$$
\lambda_{1}\left[B_{R}(x), \mu^{+}, \mu^{-}\right]<\min \left(\lambda_{V}\left[\beta l_{1}, \beta l_{2}\right], \lambda_{H}\left[\beta l_{1}, \beta l_{2}\right]\right)
$$

when $\beta$ is large. This means that the two stripe-configurations are not optimal for species persistence when $\beta$ is large and $A^{+}$is fixed.

\footnotetext{
${ }^{1}$ Actually, this property could be viewed as a consequence of Step 2 of Appendix D, since $\mu_{\beta}$ corresponds to an optimal configuration among all $L_{1}$-periodic one-dimensional configurations for which the habitat has a total length equal to $A^{+} /\left(\beta l_{2}\right)$ in $\left[0, L_{1}\right]$.
} 


\section{References}

[1] J.E.M. Baillie, C. Hilton-Taylor, S.N. Stuart, (Eds.), 2004 IUCN Red List of Threatened Species. A Global Species Assessment. IUCN, Gland, Switzerland and Cambridge, UK, 2004.

[2] A. Treves, L. Naughton-Treves, E.K. Harper, D.J. Mladenoff, R.A. Rose, T.A. Sickley, A.P. Wydeven, Predicting Human-Carnivore Conflict: a Spatial Model Derived from 25 Years of Data on Wolf Predation on Livestock, Conservation Biology 18 (1) (2004) 114-125.

[3] K. Morita, S. Yamamoto, Effects of habitat fragmentation by damming on the persistence of stream-dwelling charr populations, Conservation Biology 16 (5) (2002) 1318-1323.

[4] S.L. Pimm, H.L. Jones, J. Diamond, On the risk of extinction, Am. Nat. 132 (1988) 757-785.

[5] D. Matthies, I. Bräuer, W. Maibom, T. Tscharntke, Population size and the risk of local extinction: empirical evidence from rare plants, Oikos 105 (3) (2004) 481-488.

[6] L. Fahrig, Effects of habitat fragmentation on biodiversity, Annu. Rev. Ecol. Syst. 34 (2003) 487-515.

[7] D. Simberloff, The contribution of population and community biology to conservation science, Annu. Rev. Ecol. Syst. 19 (1988) 473-511.

[8] A.D. Chalfoun, F.R. Thompson, M.J. Ratnaswamy, Nest predators and fragmentation: a review and meta-analysis, Conservation Biology 16 (2002) 306318.

[9] T.E. Lovejoy, R.O. Bierregaard, A.B. Rylands, J.R. Malcolm, C.E. Quintela, L.H. Harper, K.S. Brown, A.H. Powell, G.V.N. Powell, H.O.R. Schubart, M. Hays, Edge and other effects of isolation on Amazon forest fragments, in: M. Soulé, (Ed.), Conservation Biology Sinauer Associates, Inc, Sunderland, MA, 1986, pp. 257-285.

[10] J.M. Diamond, The island dilemma: Lessons of modern biogeographic studies for the design of natural reserves, Biol. Conserv. 7 (1975) 129-146.

[11] K.A. With, A.W. King, Extinction thresholds for species in fractal landscapes. Conservation Biology 13 (1999) 314-326.

[12] P.M. Kareiva, N. Shigesada, Analyzing insect movement as a correlated random walk, Oecologia 56 (1983) 234-238.

[13] L.M. Marsh, R.E. Jones, The form and consequences of random walk movement models, J. Theor. Biol. 133 (1988) 113-131. 
[14] L.J. Gross, K.A. Rose, E.J. Rykiel, W. Van Winkle, E.E. Werner, Individual-based modeling, in: D.L. DeAngelis, L.J. Gross, (Eds.), Summary of a workshop. Individual-based Models and Approaches in Ecology: Populations, Communities, and Ecosystems, Routledge, Chapman and Hall, New York, 1992, pp. 511-552.

[15] K. Pearson, J. Blakeman, Mathematical Contributions of the Theory of Evolution, XV, A Mathematical Theory of Random Migration, Drapersi Company Research Mem. Biometrics Series III, Dept. Appl. Meth. Univ. College, London, 1906.

[16] R.A. Fisher, The wave of advance of advantageous genes, Ann. Eugenics 7 (1937) 355-369.

[17] A.N. Kolmogorov, I.G. Petrovsky, N.S. Piskunov, Étude de l'équation de la diffusion avec croissance de la quantité de matière et son application à un problème biologique, Bull. Univ. État Moscou, Série Internationale A 1 (1937) 1-26.

[18] J.G. Skellam, Random dispersal in theoretical populations, Biometrika 38 (1951) 196-218.

[19] P. Turchin, Quantitative Analysis of Movement: Measuring and Modeling Population Redistribution in Animals and Plants, Sinauer Associates, Sunderland, MA, 1998.

[20] J.D. Murray, Mathematical biology, Third edition, Interdisciplinary Applied Mathematics 17, Springer-Verlag, NY, 2002.

[21] A. Okubo, S.A. Levin, S.A., Diffusion and Ecological Problems - Modern Perspectives, Second edition, Springer-Verlag, 2002.

[22] N. Shigesada, K. Kawasaki, E. Teramoto, Traveling periodic waves in heterogeneous environments., Theor. Popul. Biol. 30 (1986) 143-160.

[23] R.S. Cantrell, C. Cosner, Diffusive logistic equations with indefinite weights: population models in disrupted environments, Proc. Roy. Soc. Edinb. 112 (1989) 293-318.

[24] N. Shigesada, K. Kawasaki, Biological Invasions: Theory and Practice, Oxford Series in Ecology and Evolution, Oxford University Press, Oxford, 1997.

[25] H. Berestycki, F. Hamel, L. Roques, Analysis of the periodically fragmented environment model : I - Species persistence, J. Math. Biol. 51 (1) (2005) 75113.

[26] H. Berestycki, F. Hamel, L. Roques, Analysis of the periodically fragmented environment model : II - Biological invasions and pulsating travelling fronts, J. Math. Pures Appl. 84 (8) (2005) 1101-1146. 
[27] L. Roques, R.S. Stoica, Species persistence decreases with habitat fragmentation: an analysis in periodic stochastic environments, J. Math. Biol. (2007) DOI: 10.1007/s00285-007-0076-8.

[28] D.G. Aronson, H.F. Weinberger, Multidimensional nonlinear diffusions arising in population genetics, Adv. Math. 30 (1978) 33-76.

[29] H. Berestycki, F. Hamel, Front propagation in periodic excitable media, Comm. Pure Appl. Math. 55 (2002) 949-1032.

[30] N. Kinezaki, K. Kawasaki, F. Takasu, N. Shigesada, Modeling biological invasion into periodically fragmented environments, Theor. Popul. Biol. 64 (2003) 291-302.

[31] R.S. Cantrell, C. Cosner, Spatial Ecology via Reaction-Diffusion Equations, Series In Mathematical and Computational Biology, John Wiley and Sons, Chichester, Sussex UK, 2003.

[32] J.W.S. Rayleigh, The Theory of Sound, 2nd ed. revised and elarged (in 2 vols.), Dover Publications, NY (republication of the 1894-1896 edition), 1945.

[33] G. Faber, Beweis, dass unter allen homogenen Membranen von gleicher Fläche und gleicher Spannung die kreisförmige den tiefsten Grundton gibt, S.-B. Math. Nat. Kl. Bayer Akad. Wiss. (1923) 169-172.

[34] E. Krahn, Über eine von Rayleigh formulierte Minimaleigenschaft des Kreises, Math. Ann. 94 (1925) 97-100.

[35] E. Krahn, Über Minimaleigenschaft der Kugel in drei und mehr Dimensionen. Acta Comm. Univ. Tartu (Dorpat) A9 (1926) 1-44.

[36] Ü. Lumiste, J. Peetre, Edgar Krahn, 1894-1961, A Centenary Volume, IOS Press, Amsterdam, 1994.

[37] H. Amann, Fixed point equations and nonlinear eigenvalue problems in ordered Banach spaces, SIAM Rev. 18 (1976) 620-709.

[38] H. Berestycki, Le nombre de solutions de certains problèmes semi-linéaires elliptiques, J. Func. Anal. 40 (1981) 1-29.

[39] G. Nadin, PhD Thesis, in preparation.

[40] K. Goldberg, M. Newman, E. Haynsworth, Combinatorial Analysis, in: M. Abramowitz, I.A. Stegun, (Eds.), Handbook of Mathematical Functions with Formulas, Graphs, and Mathematical Tables, 9th printing, Dover, New York, 1972, pp. 825-826.

[41] E.M. Harrell, P. Kröger, K. Kurata, On the placement of an obstacle or a well so as to optimize the fundamental eigenvalue, SIAM J. Math. Anal. 33 (1) (2001) 240-259. 
[42] S. Chanillo, D. Grieser, M. Imai, K. Kurata, I. Ohnishi, Symmetry Breaking and Other Phenomena in the Optimization of Eigenvalues for Composite Membranes, Commun. Math. Phys. 214 (2000) 315-337.

[43] E. Oudet, Numerical minimization of eigenmodes of a membrane with respect to the domain, ESAIM COCV 10 (2004) 315-335.

[44] S. Hannunen, B. Ekbom, Host plant influence on movement patterns and the subsequent distribution of Lygus rugulipennis (Heteroptera: Miridae) nymphs, Environ. Entomol. 30 (3) (2001) 517-523.

[45] J.A.G. Jaeger, L. Fahrig, Effects of Road Fencing on Population Persistence. Conservation Biology 18 (6) (2004) 1651-1657.

[46] M.D. Chekroun, L.J. Roques, Models of population dynamics under the influence of external perturbations: mathematical results, C. R. Acad. Sci. Paris, Ser. I 343 (2006) 307-310.

[47] H. Berestycki, L. Nirenberg, S.R.S. Varadhan, The principal eigenvalue and maximum principle for second order elliptic operators in general domain, Comm. Pure Appl. Math. 47 (1994) 47-92.

[48] D. Gilbarg, N.S. Trudinger, Elliptic partial differential equations of second order, Springer-Verlag, Berlin, 1983. 